\title{
Experimental Results on an Integrated GPS and Multisensor System for Land Vehicle Positioning
}

\author{
Umar Iqbal, ${ }^{1}$ Tashfeen B. Karamat, ${ }^{2}$ Aime F. Okou, ${ }^{2}$ and Aboelmagd Noureldin ${ }^{1,2}$ \\ ${ }^{1}$ Department of Electrical and Computer Engineering, Queen's University, Kingston, ON, Canada K7L 3N6 \\ ${ }^{2}$ Department of Electrical and Computer Engineering, Royal Military College of Canada, Kingston, ON, Canada K7K 7B4
}

Correspondence should be addressed to Tashfeen B. Karamat, tashfeen.karamat@rmc.ca

Received 7 October 2008; Revised 7 January 2009; Accepted 22 February 2009

Recommended by Abbas Mohammed

Global position system (GPS) is being widely used in land vehicles to provide positioning information. However, in urban canyons, rural tree canopies, and tunnels, the GPS satellite signal is usually blocked and there is an interruption in the positioning information. To obtain positioning solution during GPS outages, GPS can be augmented with an inertial navigation system (INS). However, the utilization of full inertial measurement unit (IMU) in land vehicles could be quite expensive despite the use of the microelectromechanical system (MEMS)-based sensors. Contemporary research is focused on reducing the number of inertial sensors inside an IMU. This paper explores a multisensor system (MSS) involving single-axis gyroscope and an odometer to provide full 2D positioning solution in denied GPS environments. Furthermore, a Kalman filter (KF) model is utilized to predict and compensate the position errors of the proposed MSS. The performance of the proposed method is examined by conducting several road tests trajectories using both MEMS and tactical grade inertial sensors. It was found that by using proposed MSS algorithm, the positional inaccuracies caused by GPS signal blockages are adequately compensated and resulting positional information can be used to steer the land vehicles during GPS outages with relatively small position errors.

Copyright () 2009 Umar Iqbal et al. This is an open access article distributed under the Creative Commons Attribution License, which permits unrestricted use, distribution, and reproduction in any medium, provided the original work is properly cited.

\section{Introduction}

Global positioning system (GPS) provides positioning, velocity, and time information with consistent and acceptable accuracy when there is direct line of sight to four or more satellites $[1,2]$. However, it may suffer from outages, jamming, and multipath effects in urban canyons and rural foliage canopies. Inertial navigation system (INS), on the other hand, is self-contained which is immune to external interference, but its accuracy deteriorates in the longterm due to sensor's bias, drift, scale factor instability and misalignment [3-5]. By integrating the GPS and INS signals, a complementary solution can be obtained that is often more accurate than that of each independent system [3]. For instance, GPS derived positions have approximately white noise characteristics with bounded errors and can therefore be used to update INS and improve its long-term accuracy $[4,5]$, whereas INS provides positioning information during GPS outages, assists GPS signal reacquisition after an outage, and reduces the search domain required for detecting and correcting GPS cycle slips [1-5]. INS is also capable of providing positioning, velocity, and attitude information at higher data rates than GPS. Kalman filter (KF) is traditionally used to optimally fuse the position and velocity information from both INS and GPS [6-13]. However, cost and space constraints are the two primary obstacles that have prevented the utilization of either navigation or tactical grade INS inside land vehicles or other low cost applications [14]. The recent developments of microelectromechanical systems (MEMSs) inertial sensors have enabled production of lower cost and smaller size inertial measurement units (IMUs). However, the utilization of full IMU in land vehicles and personal location systems is still relatively expensive despite the use of MEMS-based sensors. The price of the gyroscopes mostly contributes to the overall cost of an IMU. Current research trend is to investigate the applicability of reduced number of sensors inside an IMU and examine the influence of this reduction on the overall positioning accuracy [15].

This research focuses on analyzing the merits and limitations of implementing a multisensor system (MSS) using 
a single-axis gyroscope and an odometer. It also discusses the integration of GPS with INS using KF to obtain the best possible positioning solution which can fill the niche of low cost and small size systems for vehicular positioning without increasing installation complexity and compromising ease of use.

\section{Background}

Several research activities have been reported to achieve navigation solutions using alternative approaches with fewer numbers of sensors and their integration with GPS. In a gyro-free inertial measurement unit (GF-IMU) approach by Chen, accelerometers-only configuration is used to measure the three accelerations and the attitude of a rigid body in 3D space [16]. Chen developed optimal cube-like configuration of six distributed accelerometers that allows rotational acceleration to be directly calculated as a function of the accelerometer outputs. The position and velocity estimates are obtained by integration of the accelerometer data which leads to errors growth with time. Mostov et al. [17] developed a calibration method for compensating the configuration errors in Chen's scheme. However, the errors diverge quickly in case of the GPS outages. Park and Tan [18] showed an integrated GPS and GF-IMU system using KF error models to estimate the errors. However, GF-IMU diverges at a rate greater than that of gyroscope-based IMU. Another GF-IMU technique is based on Parsa et al. [19] which uses a network of four or more triaxial accelerometers for GPS/INS integration. However, Parsa's system requires long calibration and configuration procedures [20].

The problem of keeping the accuracy of the INS within bounds can also be addressed by considering the vehicle motion constraints arising from the fact that the land vehicles mostly travel on roads [21]. Previous studies show that a lateral constraint applied to wheel speed sensor integration is effective only when the vehicle operates on a flat road and no side slip occurs [22]. Brandt and Gardner [23] proposed a system based on one accelerometer and three gyroscopes. In addition, the odometer was used to provide auxiliary information about the vehicle's speed. Constraints on the motion of the land vehicles are used to derive set of navigation equations. However the proposed system mainly relies on keeping all three gyroscopes which are costly as compared to accelerometers.

To make a simple, practical, and low-cost solution, Wang [24] proposed an accelerometer and magnetometer-based system that relied on constrains defined by Brandt and Gardner [23] to reduce the navigation errors. The vehicle's velocity, obtained from the time integration of the measurements of a forward accelerometer, was compensated by a pretrained neural network. The magnetic disturbances, which are usually present in down town areas, are unpredictable and could bias the magnetometer output significantly. Even in areas where modest magnetic disturbances are present, the performance of proposed system was inversely proportional to the length of the GPS outages, which results into large errors for long GPS outages.
Recently, El-Sheimy [15] also analyzed and compared the performance of two "Partial IMU" systems which are based on (1) a single gyro and three accelerometers denoted as 1G3A and (2) a single gyro and two accelerometers (1G2A). He also applied nonholonomic constraints and odometer updates during GPS outages. His partial IMU systems promise to reduce the cost of an IMU up to $65 \%$ while maintaining reasonable navigation performance as compared to full IMU systems.

Aforementioned studies focused on reducing the cost of the navigation systems based on reducing the number of sensors. However, most of them affected the performance and others are quite complex. Earlier, the authors have also presented an algorithm for reduced sensor system [25] which only pertained to the high end tactical grade sensors. However, there is a need for a lower cost solution with MEMS grade sensors for maximum affordability. This calls for different sensor error models and subtle compromise in tuning of KF parameters which has been addressed in this paper. The results of MEMS grade sensor have been juxtaposed with high end tactical grade sensors for ready comparison.

\section{Research Objectives}

The primary objective of this research is to reduce the cost of the overall positioning system for land vehicles without appreciable performance compromise. The gyroscopes technology is what mostly contributes to the overall cost of an IMU. Hence, this research investigates the applicability of reduced number of gyroscopes inside an IMU and examines its influence on the overall positioning accuracy. A key advantage includes reduction of the gyroscope error characteristics especially when MEMS inertial sensors are used. This paper suggests a multisensor system (MSS) involving single-axis gyroscope and a speed sensor to provide full 2D positioning solution, especially in denied GPS environments. With the reasonable assumption that the vehicle mostly stays in the horizontal plane, the vehicle's speed from the speed sensor is used together with the heading information obtained from the gyroscope to determine the velocities along the East and North directions. Consequently, the vehicle's longitude and latitude are determined. MSS-based position and velocity are integrated with GPS data using KF to provide robust positioning solution. The MSS is tested on real-life road trajectories to assess its performance and results are analyzed in detail.

\section{Methodology}

4.1. Multisensor System (MSS). To cater for all the threedimensional dynamics of the vehicle, traditionally, a total of six sensors are used in a full IMU which comprise three gyroscopes and three accelerometers. However, for the reasons mentioned earlier, simpler sensor configurations are being investigated by contemporary researchers. In this paper, only one gyroscope and one odometer are used as sensor configuration to provide the positioning solution in 


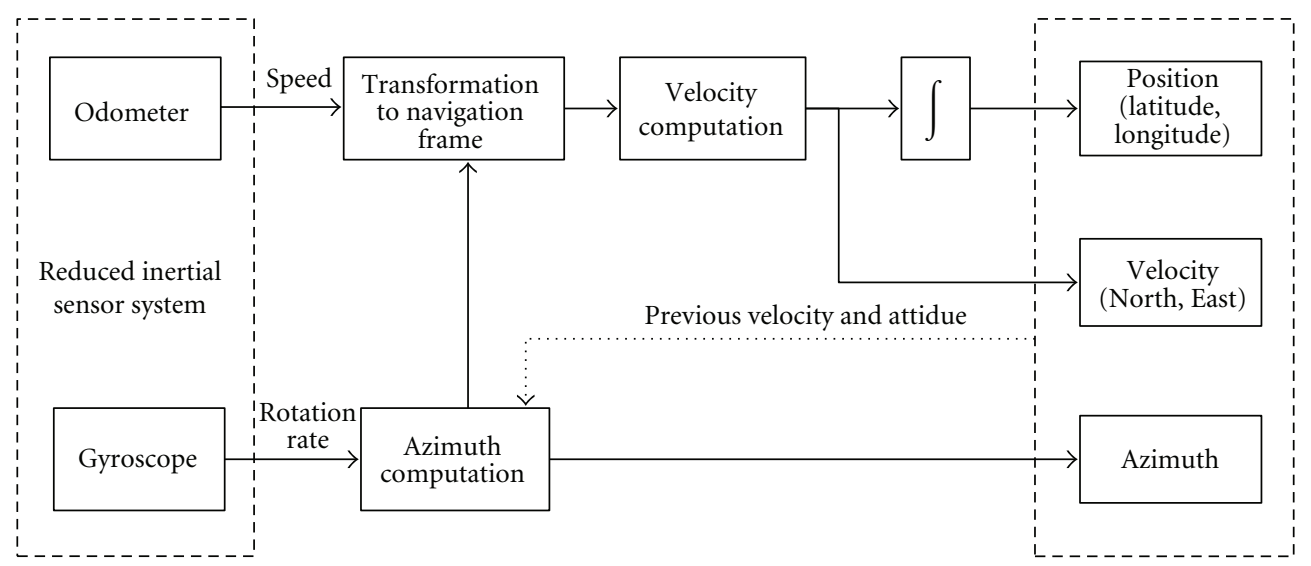

FIGURE 1: Block diagram of MSS mechanization.

the horizontal plane. The MSS mechanization is developed assuming the vehicle is mostly travelling in the horizontal plane. Thus, the vehicle's velocity along the East and North directions as well as the latitude and longitude can be obtained by measuring the vehicle speed along its forward axis and the heading angle. Therefore, one gyroscope and an odometer can be used to provide complete two-dimensional positioning solution. It is a recursive process based on initial conditions or the previous output and the new measurements. The overview of the MSS mechanization can be reviewed in Figure 1.

\section{MSS Implementation Details}

5.1. MSS Mechanization. For the MSS, one single-axis gyroscope, mounted with its sensitive axis along the vertical direction of the vehicle, is used to measure the orientation of the vehicle in horizontal plane (the azimuth angle). This gyroscope measures the rotation rate in the body frame which cannot be deduced directly in the local-level frame (LLF) for two reasons. The first reason is the component of Earth rotation rate along the vertical direction $\left(\omega^{e} \sin \varphi\right)$, where $\omega^{e}$ is the Earth rotation rate and $\varphi$ is the latitude. The second reason is of the change of orientation of the LLF with respect to earth $\left(V_{e} \tan \varphi /\left(R_{N}+h\right)\right)$, where $V_{e}$ is the velocity along the East direction, $h$ is the altitude, and $R_{N}$ is the meridian radius of curvature. Therefore, rate of change of the azimuth angle $(A)$ can be written as follows:

$$
\dot{A}=\omega_{z}-\omega^{e} \sin \varphi-\frac{V_{e} \tan \varphi}{R_{N}+h} .
$$

Velocities along the East and North directions are determined using the vehicle speed obtained from the odometer along with the heading information obtained from the gyroscope, with the assumption that the vehicle mostly stays in the horizontal plane. An odometer is a device used for indicating distance traveled by the vehicle. In modern systems, odometer is an electronic device that generates an integer number of digital pulses each time a wheel on the vehicle makes one revolution. These numbers of pulses are multiplied by an odometer scale factor to measure the distance traveled by the vehicle in a specific time interval [26]. The vehicle speed can then be obtained from the odometer measurements. Some errors contribute to the odometer measurement accuracy. For instance, the odometer scale factor depends on the radius of the vehicle's wheel, which can change with tire pressure, temperature, tread wear, and speed. Wide-ranging sources and analysis of error for speed and odometer measurements are available in the literature [27, 28]. The MSS mechanization process is summarized in a detailed block diagram in Figure 2.

To obtain MSS positional information in the East and North directions, the initial heading (azimuth angle $A$ ) must be known. In this study, we rely on the GPS driven azimuth which is computed using the GPS east and north velocities once the vehicle starts to move to give the initial azimuth angle for the MSS mechanization. The change in the azimuth angle is then obtained by numerically integrating (1). The computed azimuth allows the transformation of the vehicle's speed along the forward direction $V_{o d}$ (obtained from the odometer measurements) to east and north velocities as follows:

$$
V=\left(\begin{array}{c}
V_{e} \\
V_{n}
\end{array}\right)=\left(\begin{array}{cc}
V_{\mathrm{od}} & \sin A \\
V_{\mathrm{od}} & \cos A
\end{array}\right) .
$$

The east and north velocities are transformed into geodetic coordinates and then integrated over the sample interval to obtain positions in latitude $(\phi)$ and longitude $(\lambda)$ as per the following equation:

$$
\dot{\mathrm{r}}=\left(\begin{array}{c}
\dot{\varphi} \\
\dot{\lambda}
\end{array}\right)=\left(\begin{array}{cc}
0 & \frac{1}{R_{M}+h} \\
\frac{1}{\left(R_{N}+h\right) \cos \varphi} & 0
\end{array}\right)\left(\begin{array}{c}
V_{e} \\
V_{n}
\end{array}\right) .
$$

5.2. Overview of Kalman Filter. Kalman filtering is an optimal estimation tool that provides a sequential recursive algorithm for the optimal least mean variance (LMV) estimation of the system states [29]. In addition to its benefits as an optimal estimator, the KF provides real-time statistical data related to the estimation accuracy of the system states, which is very useful for quantitative error analysis [7]. The 


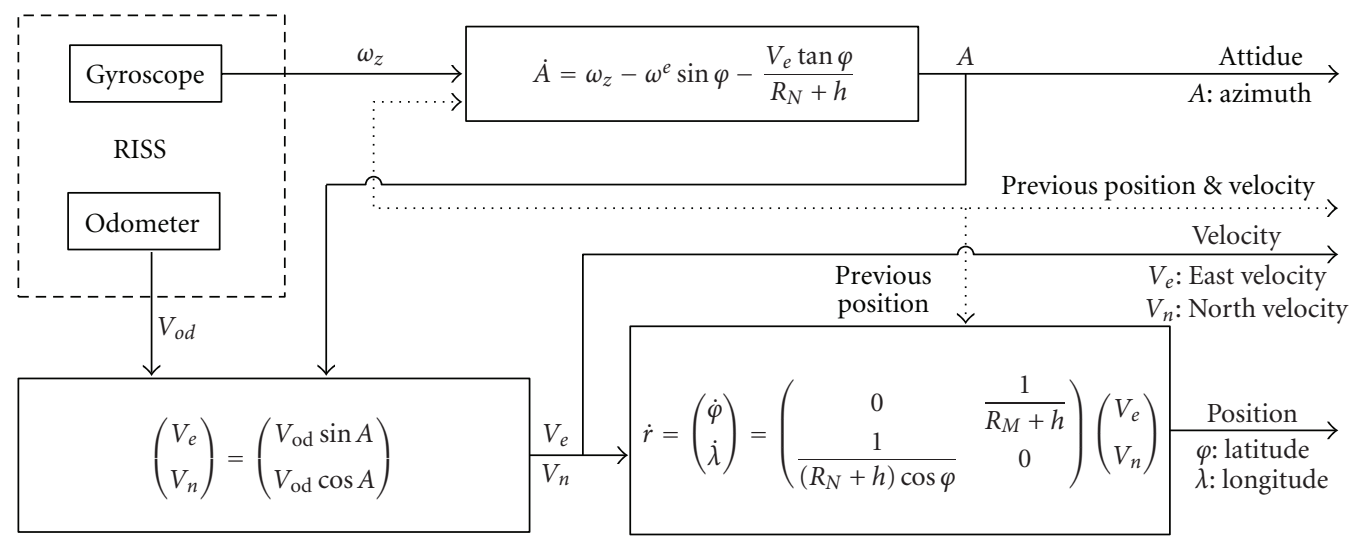

Figure 2: Detailed block diagram of MSS mechanization.

filter generates its own error analysis with the computation of the error covariance matrix, which gives an indication of the estimation accuracy.

5.2.1. Discrete Linearized Kalman Filter. Since we are dealing with nonlinear system and the low-cost MEMS inertial sensors are of relatively poor performance, it was therefore decided to use linearized KF (LKF) where nonlinear systems are linearized about the known trajectory (which, in our case, is output of the mechanization) and then well-known $\mathrm{KF}$ equations are applied to the linearized equations. This is done through the open-loop (feedback) implementation of INS/GPS integration as shown in Figure 3. The theory of KF is well established and details can be found in [6-10, 12, 13]; however, a brief review of the linearization process is in order.

The discrete time nonlinear dynamic model state equation is given as follows [8]:

$$
x_{k}=f\left(x_{k-1}, k-1\right)+g\left(x_{k-1}, k-1\right) w_{k-1}, \quad w_{k} \sim N\left(0, Q_{k}\right) .
$$

The nonlinear measurement equation is

$$
z_{k}=h\left(x_{k}, k\right)+v_{k}, \quad v_{k} \sim N\left(0, R_{k}\right) .
$$

If $\tilde{x}_{k-1}$ is a known (or nominal) trajectory of the system, then we can write the equation of the system (neglecting the noise) as follows:

$$
\tilde{x}_{k}=f\left(\tilde{x}_{k-1}, k-1\right) .
$$

An arbitrary trajectory of the system described by (4) can be described as follows:

$$
x_{k-1}=\tilde{x}_{k-1}+\Delta x_{k-1},
$$

where $\Delta x_{k-1}$ is the deviation of $x_{k-1}$ from $\tilde{x}_{k-1}$. By these definitions, (4) can be represented as follows:

$$
\tilde{x}_{k}+\Delta x_{k}=f\left(\tilde{x}_{k-1}+\Delta x_{k-1}, k-1\right)+g\left(\tilde{x}_{k-1}+\Delta x_{k-1}, k-1\right) w_{k-1} \text {. }
$$

Applying Taylor's expansion about $\tilde{x}_{k-1}$ and neglecting higher order terms, we have

$$
\tilde{x}_{k}+\Delta x_{k} \approx f\left(\tilde{x}_{k-1}, k-1\right)+\frac{\partial f}{\partial x}\left(\tilde{x}_{k-1}\right) \Delta x_{k-1}+g\left(\tilde{x}_{k-1}, k-1\right) w_{k-1} .
$$

From definition of (6), (9) can be written as follows:

$$
\Delta x_{k} \approx \frac{\partial f}{\partial x_{k-1}}\left(\tilde{x}_{k-1}, k-1\right) \Delta x_{k-1}+g\left(\tilde{x}_{k-1}, k-1\right) w_{k-1} .
$$

Now, (5) can be expressed as follows:

$$
z_{k}=h\left(\tilde{x}_{k}+\Delta x_{k}, k\right)+v_{k} .
$$

By using Taylor's expansion about $\tilde{x}_{k}$,

$$
z_{k} \approx h\left(\tilde{x}_{k}, k\right)+\frac{\partial h}{\partial x}\left(\tilde{x}_{k}, k\right) \Delta x_{k}+v_{k}
$$

By letting $\Phi_{k-1}=\partial f\left(\hat{x}_{k-1}, k-1\right) / \partial x_{k-1}, G_{k-1} \triangleq g\left(\tilde{x}_{k-1}, k-\right.$ $1), H_{k} \triangleq(\partial h / \partial x)(\tilde{x}, k)$, and $y_{k} \triangleq z_{k}-h\left(\tilde{x}_{k}, k\right),(10)$ and (12) can be written as follows:

$$
\begin{aligned}
\Delta x_{k} & \approx \Phi_{k-1} \Delta x_{k-1}+G_{k-1} w_{k-1}, \\
y_{k} & \approx H_{k} \Delta x_{k}+v_{k} .
\end{aligned}
$$

Now, the KF equations can be applied to this linearized state space model to obtain (LMV) estimate $\Delta \hat{x}_{k}$ of $\Delta x_{k}$ based on $y_{1}, \ldots, y_{k}$. Estimate of state $x_{k}$ can now be calculated by using

$$
\hat{x}_{k}=\tilde{x}_{k}+\Delta \hat{x}_{k}
$$

A summary of KF equations is summarized in Table 1.

5.3. Kalman Filter for GPS/MSS Data Fusion. KF used in this study operates in a loosely coupled fashion to fuse the MSS computed position and velocity components along the horizontal channels with the corresponding GPS positions and velocities. This enables the computation of the MSS positions, velocities, and azimuth errors as well as the 


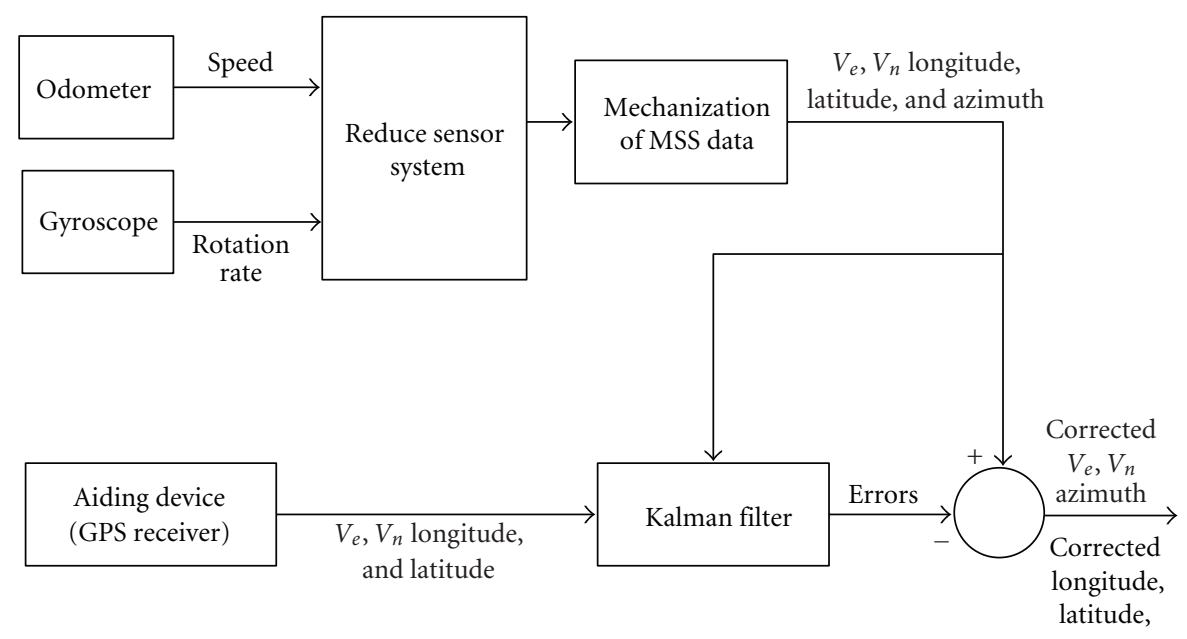

FIgURE 3: Kalman filter for MSS/GPS integration.

TABLE 1: Summary of Kalman filter equations.

Summary of Kalman filter equations

Linear perturbation prediction:

$$
\Delta \hat{x}_{k}^{-}=\Phi_{k-1} \Delta \hat{x}_{k-1} \text {. }
$$

Conditioning the predicted perturbation on the measurement:

Predicted measurement:

$$
\Delta \hat{x}_{k}=\Delta \hat{x}_{k}^{-}+K_{k}\left(y_{k}-\hat{y}_{k}^{-}\right) \text {. }
$$

A priori covariance matrix:

$$
\hat{y}_{k}^{-}=H_{k} \Delta \hat{x}_{k-1}^{-} \text {. }
$$

Kalman gain:

$$
P_{k}^{-}=\Phi_{k-1} P_{k-1} \Phi_{k}^{T}+G_{k-1} Q_{k-1} G_{k-1}^{T} .
$$

A posteriori covariance matrix:

$$
K_{k}=\left(P_{k}^{-}\right) H_{k}^{T}\left(H_{k} P_{k}^{-} H_{k}^{T}+R_{k}\right)^{-1} .
$$

$$
P_{k}=\left(I-K_{k} H_{k}\right) P_{k}^{-}\left(I-K_{k} H_{k}\right)^{T}+K_{k} R_{k} K_{k}^{T} .
$$

gyroscope and odometer residual random errors. A block diagram of the MSS and GPS integration is shown in Figure 3.

KF outputs two important variables, the estimated state vector $x$ and the covariance matrix $P$. The estimated state vector is a vector of MSS positions, velocities, and azimuth errors augmented with the sensors random errors (for both gyroscope and the odometer driven acceleration). The covariance matrix is a measure of the estimation uncertainty which takes into consideration how the sensor noise and dynamic uncertainty contribute to the uncertainty about the estimated error states [30]. By maintaining an estimate of its own estimation uncertainty and the relative uncertainty in the sensor outputs, the KF is able to optimize the estimate to minimize the estimation error. The measurements update (GPS position and velocity) may be corrupted with additive zero-mean white noise. In order for a KF to produce a statistically optimal estimate of its state, the filter's model equations, measurement equations, and spectral density matrices should accurately describe the dynamical and statistical properties of the system of interest.
5.3.1. MSS Error Model. The error state vector of the MSS error model includes coordinate errors $(\delta \varphi, \delta \lambda)$, velocity errors $\left(\delta V_{e}, \delta V_{n}\right)$, azimuth error $(\delta A)$, and residual random errors of odometer driven acceleration $\delta a_{o d}$ and gyroscope $\delta \omega_{z}$ as follows:

$$
x=\left(\begin{array}{lllllll}
\delta \varphi & \delta \lambda & \delta V_{e} & \delta V_{n} & \delta A & \delta a_{o d} & \delta \omega_{z}
\end{array}\right)^{T}
$$

These errors are passed from one estimate to another with the overall uncertainty in the precision of the estimated quantity drifting with time $[6,12]$. Therefore, error models are required for analysis and estimation of different error sources associated with the proposed MSS.

Since the errors in dynamic systems are function of time, they are described by differential equations [31]. Linearization of a nonlinear dynamic system is the most common approach to derive a set of linear differential equations that define the error states of a dynamic system $[4,32]$. Thus, the time rate of change of the MSS position errors can be derived from following equations:

$$
\left(\begin{array}{c}
\delta \dot{\varphi} \\
\delta \dot{\lambda}
\end{array}\right)=\left(\begin{array}{cc}
0 & \frac{1}{R_{M}+h} \\
\frac{1}{\left(R_{N}+h\right) \cos \varphi} & 0
\end{array}\right)\left(\begin{array}{l}
\delta V_{e} \\
\delta V_{n}
\end{array}\right)
$$

Similarly, time rate of change of the MSS velocity errors can be obtained starting from (2) and written as follows:

$$
\left(\begin{array}{c}
\delta \dot{V}_{e} \\
\delta \dot{V}_{n}
\end{array}\right)=\left(\begin{array}{cc}
\sin A & a_{\mathrm{od}} \cos A \\
\cos A & -a_{\mathrm{od}} \sin A
\end{array}\right)\left(\begin{array}{c}
\delta a_{\mathrm{od}} \\
\delta A
\end{array}\right)
$$

where $a_{\mathrm{od}}$ is the odometer driven acceleration, and $\delta a_{\mathrm{od}}$ is the corresponding residual random error. It should be highlighted that in obtaining (22), the time rate of change of the azimuth $\dot{A}$ is neglected. This is one of the limitation of the proposed method and the accuracy may deteriorate during the sharp turns of the vehicle. 
The azimuth errors mostly depend on the gyroscope residual random error (mostly due to the residual long term drifts) and can be written as follows:

$$
\delta \dot{A}=\delta \omega_{z} .
$$

5.3.2. Stochastic Modeling of Inertial Sensor Errors. In most $\mathrm{KF}$ implementations, the sensor noise portion of the state transition matrix $F$ is based on a 1st-order Gauss-Markov (GM) model. The GM process is frequently used in engineering applications because of its simplicity and partly because the designer often lacks the detailed knowledge of the process under consideration that is needed to devise a more complicated model [33].

5.3.3. The Gauss-Markov Process. A stationary Gaussian process $x(t)$ that has an exponential autocorrelation is called a Gauss-Markov (GM) process [29]. The statistics of a stationary GM process are completely described by its autocorrelation function as follows:

$$
R_{x x}(\tau)=\sigma^{2} e^{-\beta|\tau|},
$$

where $\sigma^{2}$ is the variance of the sensor noise, and $\beta$ is the reciprocal of the correlation time which corresponds to decaying exponential autocorrelation sequence. follows:

First-order Gauss-Markov process $x(t)$ is described as

$$
\dot{x}(t)=-\beta x(t)+\sqrt{2 \sigma^{2} \beta} w(t),
$$

where $w(t)$ is modeled as unity variance white Gaussian noise. A typical autocorrelation plot of a first- order GM process is shown in Figure 4. By computing the autocorrelation function, the variance $\left(\sigma^{2}\right)$ can be determined from the maximum value and the correlation time $(\tau)$ can be calculated from the time corresponding to $(1 / e) \sigma^{2}$. These parameters are determined from actual experimental data from a stationary run. In discrete-time domain, (20) is written as follows $[6,34]$ :

$$
x_{k}=(I-\beta \Delta t) x_{k-1}+\sqrt{2 \beta \sigma^{2}} w_{k} \Delta t,
$$

where $t \Delta$ is the sampling interval.

5.3.4. Gauss-Markov Model for GPS/MSS Data Fusion. In this study, the errors characteristics of both the odometer and gyrocope were observed by obtaining the autocorrelation sequence of the static data. It was noticed that the autocorrelaton of the gryroscope resembled that of first GM process. The noise from odometer was observed to be white and its autocorrelaton showed a peak around zero with some power at other frequencies. Therefore, the odometer errors were also modeled by first-order GM process with a short correlation time. These error models can be written as follows $[6,29]$ :

$$
\left(\begin{array}{c}
\delta \dot{a}_{\mathrm{od}} \\
\delta \dot{\omega}_{z}
\end{array}\right)=\left(\begin{array}{cc}
-\gamma_{\mathrm{od}} & 0 \\
0 & -\beta_{z}
\end{array}\right)\left(\begin{array}{c}
\delta a_{\mathrm{od}} \\
\delta \omega_{z}
\end{array}\right)+\left(\begin{array}{c}
\sqrt{2 \gamma_{\mathrm{od}} \sigma_{\mathrm{od}}^{2}} \\
\sqrt{2 \beta_{z} \sigma_{z}^{2}}
\end{array}\right) w(t),
$$

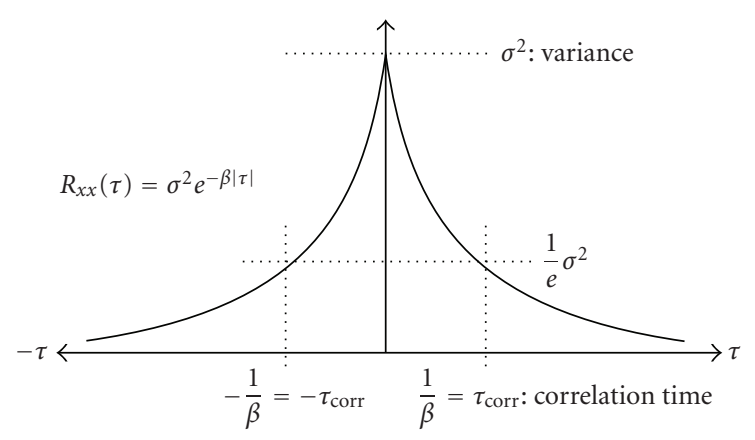

FIGURE 4: The autocorrelation sequence of a 1st-order GaussMarkov process.

where $\beta_{z}$ is the reciprocal of the correlation time of the random process associated with the gyroscope measurement, $\sigma_{z}$ is the standard deviation of this random process, and $w(t)$ is unity variance white Gaussian noise. Similarly, $\gamma_{\mathrm{od}}$ is the reciprocal of the correlation time of the random process associated with the odometer driven acceleration; $\sigma_{\text {od }}$ is the standard deviation of this random process.

\subsection{Kalman Filter Implimentation for MSS/GPS Data Fusion.}

The random processes associated with the components of the error state vector $x$ were represented by a group of firstorder state equations, which can be augmented together and described by the following discrete linear state equation:

$$
x_{k}=F_{k, k-1} x_{k-1}+G_{k-1} w_{k-1},
$$

where $x_{k}$ is the error state vector $x$ at time $t_{k}$,

$$
x_{k}=\left(\begin{array}{lllllll}
\delta \varphi & \delta \lambda & \delta V_{e} & \delta V_{n} & \delta A & \delta a_{\mathrm{od}} & \delta \omega_{z}
\end{array}\right)^{T} .
$$

$F_{k, k-1}$ is a dynamic matrix relating $x_{k-1}$ to $x_{k} ; w_{k-1}$ is a random forcing function which can be regarded as unityvariance white Gaussian noise with $G_{k-1}$ being its coefficient vector [32]. Dynamic coefficient matrix $F_{k, k-1}$ and noise coupling matrix $G_{k-1}$ can be written using 1st-order Taylor series approximation as follows:

$$
\begin{aligned}
F_{k, k-1} & =I+F \Delta t, \\
G_{k-1} & =G \Delta t .
\end{aligned}
$$

For MSS system error equations, the dynamic matrix $F$ can be written as follows:

$$
F=\left(\begin{array}{ccccccc}
0 & 0 & 0 & \frac{1}{R_{M}+h} & 0 & 0 & 0 \\
0 & 0 & \frac{1}{\left(R_{N}+h\right) \cos \varphi} & 0 & 0 & 0 & 0 \\
0 & 0 & 0 & 0 & a_{\mathrm{od}} \cos A & \sin A & 0 \\
0 & 0 & 0 & 0 & -a_{\mathrm{od}} \sin A & \cos A & 0 \\
0 & 0 & 0 & 0 & 0 & 0 & 1 \\
0 & 0 & 0 & 0 & 0 & -\gamma_{\mathrm{od}} & 0 \\
0 & 0 & 0 & 0 & 0 & 0 & -\beta_{z}
\end{array}\right)
$$


and $G$ is noise coupling matrix for the MSS dynamic model and is written as follows:

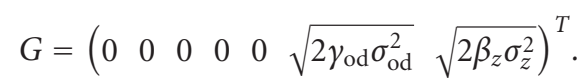

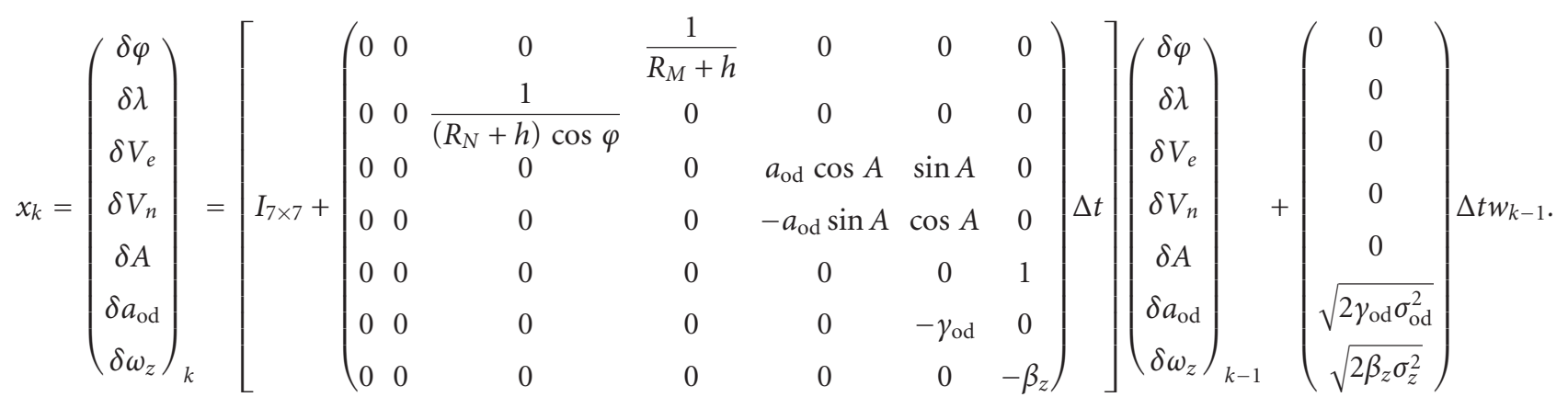

Finally the MSS error model in discrete time domian can be expressed as follows:

In order to provide optimal estimation of the above error state vecotor $x_{k}$, observations for the above discrete system can be provided in the following form:

$$
y_{k}=H x_{k}+v_{k}
$$

where $y_{k}$ is the observations vector at time $t_{k}$ giving the difference between the MSS and GPS positions and velocities, $H_{k}$ is the design matrix giving the ideal noiseless relationship between the observations vector and the state vector, and $v_{k}$ is the vector of observations random noise, which is assumed to be white sequence not correlated with the MSS system noise $w_{k}$ (i.e., $E\left(w_{k}, v_{k}^{T}\right)=0$ ). For the MSS proposed in this study, the parameters of the measurement model are given as follows:

$$
\begin{aligned}
& y=\left(\begin{array}{c}
\varphi^{\mathrm{MSS}}-\varphi^{\mathrm{GPS}} \\
\lambda^{\mathrm{MSS}}-\lambda^{\mathrm{GPS}} \\
V_{e}^{\mathrm{MSS}}-V_{e}^{\mathrm{GPS}} \\
V_{n}^{\mathrm{MSS}}-V_{n}^{\mathrm{GPS}}
\end{array}\right), \\
& H=\left(\begin{array}{lllllll}
1 & 0 & 0 & 0 & 0 & 0 & 0 \\
0 & 1 & 0 & 0 & 0 & 0 & 0 \\
0 & 0 & 1 & 0 & 0 & 0 & 0 \\
0 & 0 & 0 & 1 & 0 & 0 & 0
\end{array}\right) .
\end{aligned}
$$

So observations for the above discrete system can be written as follows:

$$
y_{k}=\left(\begin{array}{c}
\varphi^{\mathrm{MSS}}-\varphi^{\mathrm{GPS}} \\
\lambda^{\mathrm{MSS}}-\lambda^{\mathrm{GPS}} \\
V_{e}^{\mathrm{MSS}}-V_{e}^{\mathrm{GPS}} \\
V_{n}^{\mathrm{MSS}}-V_{n}^{\mathrm{GPS}}
\end{array}\right)_{k}
$$

$$
=\left(\begin{array}{lllllll}
1 & 0 & 0 & 0 & 0 & 0 & 0 \\
0 & 1 & 0 & 0 & 0 & 0 & 0 \\
0 & 0 & 1 & 0 & 0 & 0 & 0 \\
0 & 0 & 0 & 1 & 0 & 0 & 0
\end{array}\right)\left(\begin{array}{c}
\delta \varphi \\
\delta \lambda \\
\delta V_{e} \\
\delta V_{n} \\
\delta A \\
\delta a_{\mathrm{od}} \\
\delta \omega_{z}
\end{array}\right)_{k}
$$

5.4.1. Kalman Filter Parameter Tuning. For the proper implementation of $\mathrm{KF}$ and to avoid divergence, some KF parameters must be properly tuned which include initial values of error covariance matrix $P$, process noise covariance matrix $Q$, and measurment noise covariance matrix $R$. Initial values of $P$ determine the initial transient response of the filter whereas $Q$ and $R$ matrices dictate long-term performance of the filter. The tuning process is akin to numerical optimizaton problem which can be sovled by autmatic search methods or by manual calculation. Manual optimization is usually preferred [35] which has been used in this study. In manual optimizaton, an initial estimate of measurment noise covariance is obtained through offline measurments, which is adjusted untill performance of the filter no longer improves. Process noise covariance is difficult to estimate as, typically, the process cannot be observed directly [36]. Therefore, a very small initial value of process noise is injected which is gradually increased to enhance the filter performance. 
For autmatic tuning of the above mentionied paramters, sensivity analysis is perfromed and reader is refered to [35] for a detailed account. Adaptive (self-learning) filtering techqniques are also used for filter tuning where the estimated ouput of the filter is adjusted to adapt to the incoming measurments in such a way that errors in the system model are minimized [8]. Some nifty KF tuning technniques have been addressed in recent work which can be found in [3739].

5.4.2. Operation of KF during Prediction and Update Mode. In this implementation, KF normally operates in update mode where prediction of position state $\Delta \hat{x}_{k}^{-}$is updated with the GPS measurement based on gain $K$ as per Kalman gain equation in Table 1 . The gain $K$ largely depends on the tuned value of $R$ matrix and $P$ matrix as depicted in Kalman gain equation in Table 1 . The measurement error covariance matrix $R$ represents noise in the GPS measurements. The diagonal elements of $(R)$ correspond to variances of the GPS measurements

$$
R=\left(\begin{array}{cccc}
\sigma_{\varphi}^{2} & 0 & 0 & 0 \\
0 & \sigma_{\lambda}^{2} & 0 & 0 \\
0 & 0 & \sigma_{V_{e}}^{2} & 0 \\
0 & 0 & 0 & \sigma_{V_{n}}^{2}
\end{array}\right)
$$

The values of $R$ must be adjusted until optimal performance is reached by examining the diagonal elements of the covariance matrix $P$. Each KF is designed for a specific model and a specific GPS where the values are determined for optimal performance. If the diagonal elements of $R$ were set to very small values (extremely low levels of uncertainty on the GPS outputs), the KF would rely on the GPS update more than the MSS error model. On the other hand, if the diagonal elements were given large values (corresponding to high levels of GPS uncertainty), the KF would rely more on the MSS error model. Therefore, the values of the $R$ matrix should be chosen to provide balance in the system accuracy when the GPS signal is available. Other methods to tune $R$ involve receiving the dynamic GPS standard deviations directly from the GPS receiver and then scaling them for proper operation. This option is not always available since the GPS standard deviations cannot always be obtained and estimating them can introduce unwanted errors.

In case of GPS outage, theoretically, gain $K$ is reduced to zero as $R$ amounts to infinity; meaning thereby, there is no update and KF relies only on its prediction, based on the MSS error model and stochastic models of gyro and odometer residual random errors. On the availability of GPS signal, KF will resume its update stage.

\section{Experimental Work}

6.1. Equipment Set-Up. The experimental data collection was carried out using a full size General Motors passenger van carrying a suite of measurement equipment that included inertial sensors, GPS receivers, antennae, and

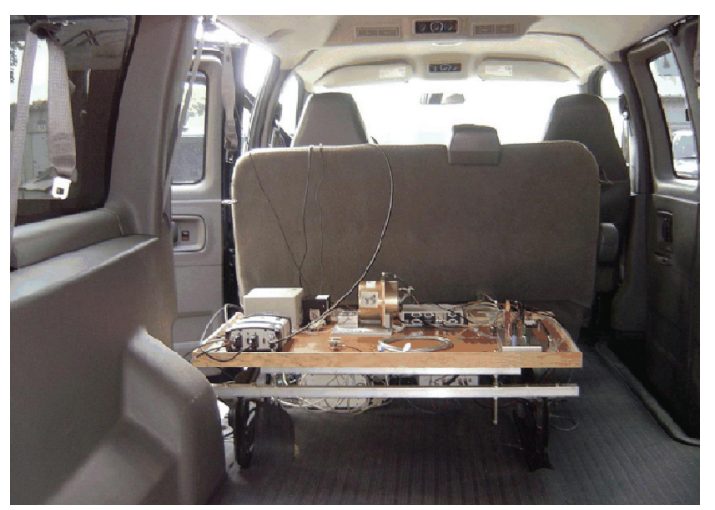

FIGURE 5: Equipment mounted inside the road test vehicle.

computers to control the instruments and acquire the data. To ease quick set-up and removal, the rear seat of the van was removed and replaced with a table that securely mounts to the seat rails and houses all the measurement equipment and accessories. A photograph of the experimental set-up is provided in Figure 5.

The inertial sensors used for this research were the MEMS grade Crossbow IMU300CC and the Honeywell HG1700 AG11 tactical grade IMU. Table 2 summarizes the physical and operating characteristics of each of the sensors. The Crossbow IMU is a six degree of freedom inertial system that uses solid state devices to measure angular rate and linear acceleration. The three angular rate sensors are bulk micromachined vibratory MEMS sensors that make use of Coriolis force to measure angular rate independent of acceleration [40]. The three accelerometers are surface micromachined silicon devices that employ differential capacitance to sense acceleration. The Honeywell HG1700 is a high end tactical grade IMU that measures angular rate and linear acceleration using three ring-laser gyroscopes and three accelerometers mounted orthogonally. The odometer data was collected using CarChip E/X (8225) data logger [41] which connects with car's ODBII interface.

Apart from providing the tactical grade raw data for the experiments, the Honeywell IMU was integrated with Novatel GPS receiver using an off the shelf assembly, the G2 Pro-Pack Span unit, also developed by Novatel. The Novatel units provide a tightly coupled INS/GPS navigation solution, which is used as a reference to compare the performance and the effectiveness of the proposed methods when applied to MEMS-based sensors.

6.2. Trajectory Data Acquisition. Data collection was accomplished using a laptop computer (Dell Notebook, $2.2 \mathrm{GHz}$, Core Duo Processor, 2GB RAM) for storing data and running the software interfaces for the instruments. The Crossbow's GYRO-VIEW software was used for the Crossbow IMU data acquisition through RS-232 connection. For the collection of tactical grade data, NovAtel CDU software was used which uses USB connection for data transfer. 


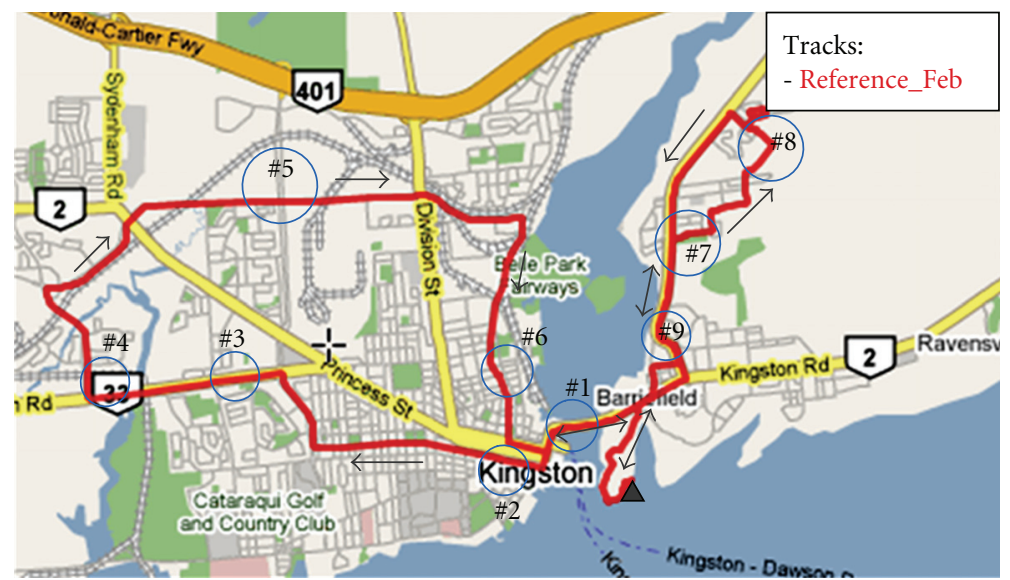

FIGURE 6: Map of trajectory no. 1 with location of GPS outages encircled.

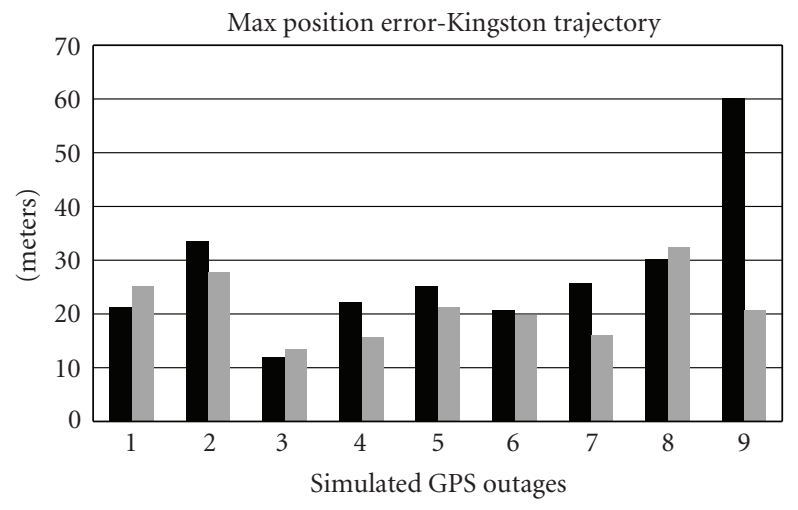

MEMS MSS

Tactical MSS

FIGURE 7: Maximum horizontal position error during nine 60second GPS outages for MEMS MSS and tactical MSS (trajectory no. 1).

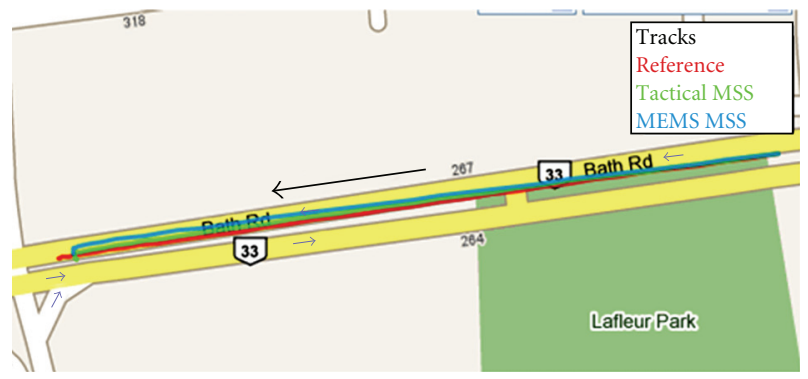

FIGURE 8: Performance during GPS outage no. 3 of trajectory no. 1.

A total of three trajectories were used in the analysis of the algorithm and the results of these trajectories are presented in the ensuing sections. These trajectories include all the real-life scenarios encountered by a typical land vehicle which include high speed highway sections, suburban roads with hills, trees and winding turns, and urban streets with frequent stops, and sudden accelerations and decelerations.
TABLE 2: Characteristics of Crossbow and Honeywell IMUs [42].

\begin{tabular}{lcc}
\hline & Crossbow IMU300CC & HG1700 \\
\hline Size & $7.62 \times 9.53 \times 3.2(\mathrm{~cm})$ & $15 \times 15 \times 10(\mathrm{~cm})$ \\
Weight & $0.59 \mathrm{~kg}$ & $0.725 \mathrm{~kg}$ \\
Max data rate & $200 \mathrm{~Hz}$ & $100 \mathrm{~Hz}$ \\
Start-up time & $<1 \mathrm{~s}$ & $<0.8 \mathrm{~s}$ \\
\hline Accelerometer & & $\pm 50 \mathrm{~g}$ \\
\hline Range & $\pm 2 \mathrm{~g}$ & $1.0 \mathrm{mg}$ \\
Bias & $\pm 30 \mathrm{mg}$ & $300 \mathrm{ppm}$ \\
Scale factor & $<1 \%$ & $0.0198 \mathrm{~m} / \mathrm{s} / \mathrm{hr}^{1 / 2}$ \\
Random walk & $<0.15 \mathrm{~m} / \mathrm{s} / \mathrm{hr}^{1 / 2}$ & \\
\hline Angular rate & & $1 \% 0 \mathrm{~g}$ \\
\hline Range & $\pm 100 \mathrm{~g}$ & $150 \mathrm{hpm}$ \\
Bias & $< \pm 2.0^{\circ} / \mathrm{s}$ & $0.125^{\circ} / \mathrm{hr}^{1 / 2}$ \\
Scale factor & $<1 \%$ & $\pm 5 \mathrm{~V} \mathrm{dc}$ \\
Random walk & $<2.25^{\circ} / \mathrm{hr}^{1 / 2}$ & $<8 \mathrm{~W}$ \\
\hline Electrical & & $\mathrm{RS}-422$ \\
\hline Input voltage & $9-30 \mathrm{~V} \mathrm{dc}$ &
\end{tabular}

The start and end points of the trajectories almost coincide and are shown by a solid triangle. The direction of the trajectory is indicated by solid arrows. Blue circles depict the place of simulated GPS outage and their radii indicate the relative length of the distance covered by the vehicle during the outage.

\section{Test Results and Discussion}

7.1. Results for Trajectory No. 1. The first trajectory was completed on the 6th of February, 2007 in the city of Kingston. The experiment was completed within the city speed limits; therefore, the speed of the vehicle was limited to $60 \mathrm{~km} / \mathrm{h}$. Duration of the trip was around 60 minutes and it started and ended at Royal Military College of Canada. The 


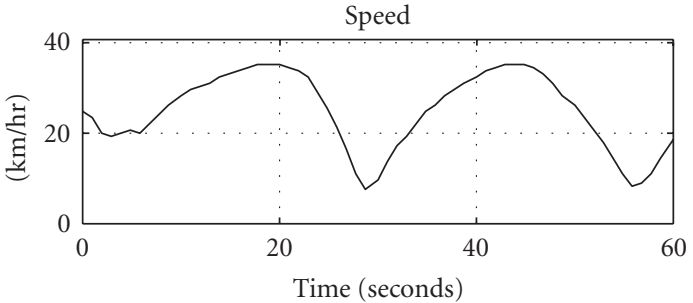

(a)

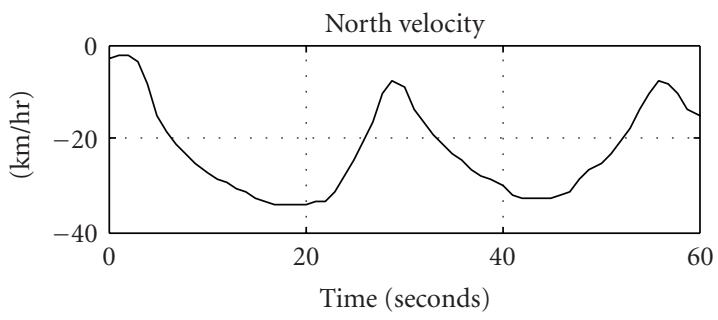

(c)

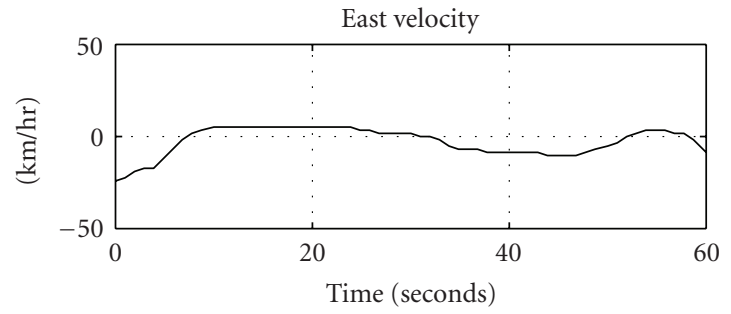

(e)

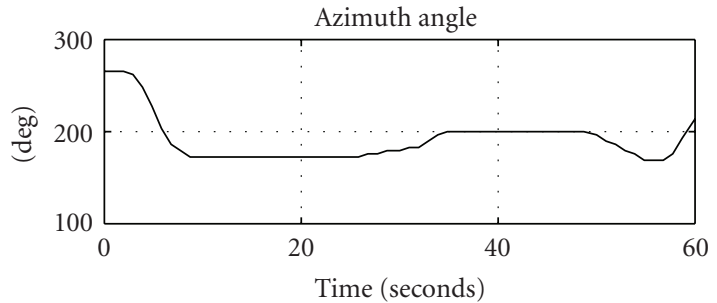

(b)

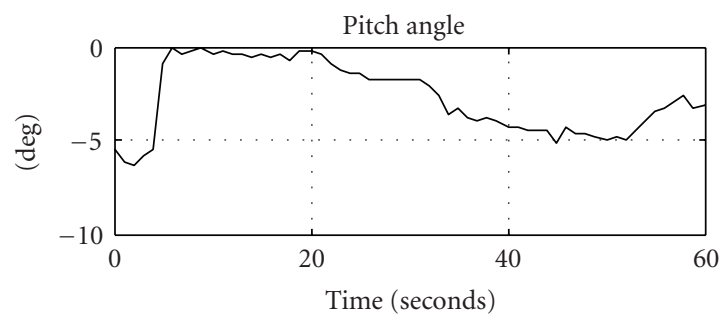

(d)

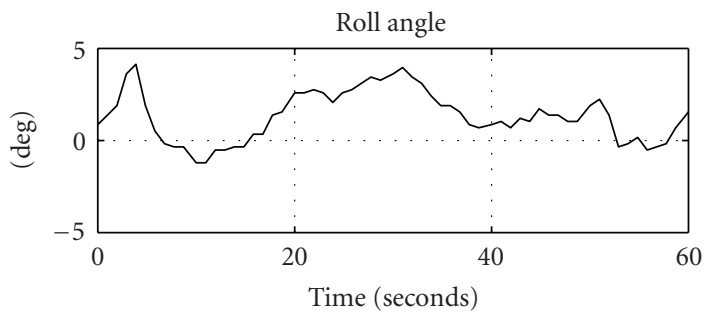

(f)

Figure 9: Details of vehicle dynamics during GPS outage no. 9 of trajectory no. 1.

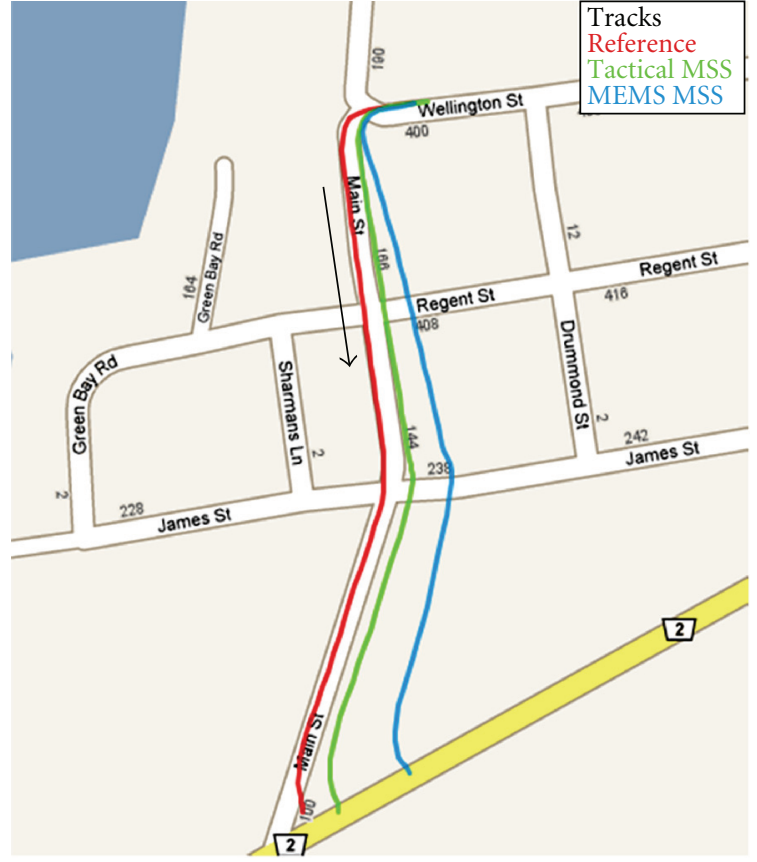

FIGURE 10: Performance during GPS outage no. 9 of trajectory no. 1. trajectory encountered numerous complete stops, resulting in many points of accelerations and decelerations. Figure 6 shows the reference solution of the trajectory with the location of the nine intentionally introduced GPS outages encircled in blue. The intentionally introduced GPS outages are such that they encompass all conditions of a typical trip including straight portions, turns, slopes, high speed, slow speeds, and stops.

Figure 7 compares MEMS MSS and tactical MSS model for nine GPS outages of 60 seconds. It was observed that, during periods of GPS outages, adequate positioning accuracy was obtained when using the MSS module. The worst accuracy took place during the GPS outage no. 9 where the maximum error reached $60 \mathrm{~m}$ with RMS of $38 \mathrm{~m}$ for MEMS MSS and maximum error for tactical MSS was only $21 \mathrm{~m}$ with an RMS of $15 \mathrm{~m}$.

The superior performance of MSS is due to odometer that has more stable bias and scale factor performance that do not drift significantly over time. In addition, MSS utilizes only one inertial sensor (gyroscope) instead of six used in a full IMU.

The best performance of the MEMS MSS was in GPS outage no. 3 and Figure 8 shows the section of the trajectory during this outage. During GPS outage no. 3, the vehicle was on relatively straight portion of the trajectory. Maximum 


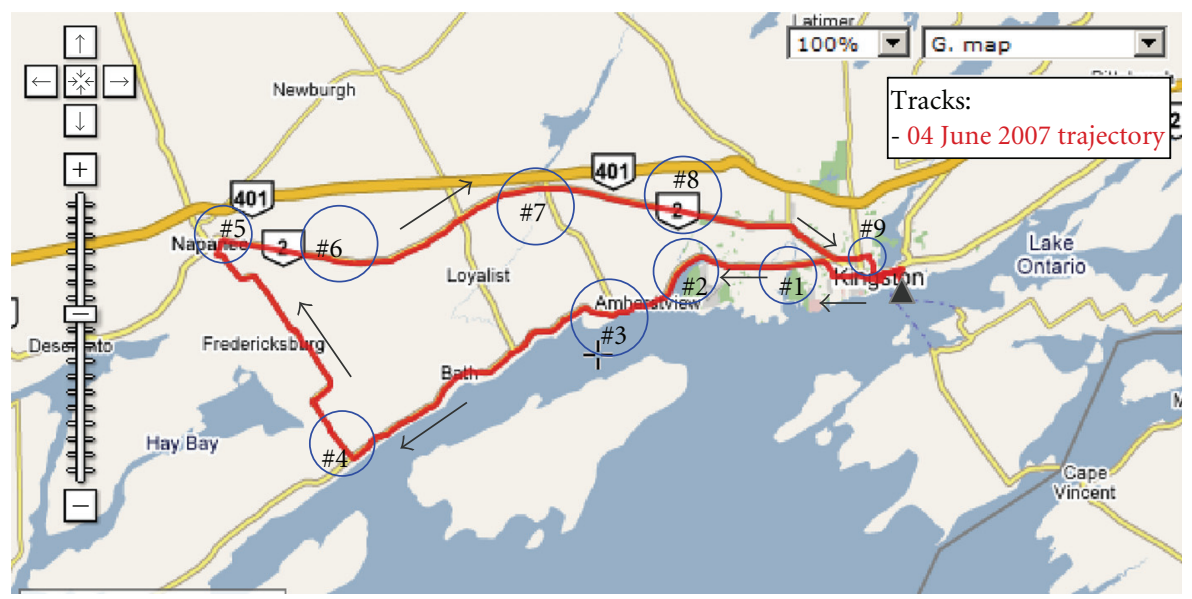

FIGURE 11: Map of trajectory no. 2 with location of nine GPS outages encircled.

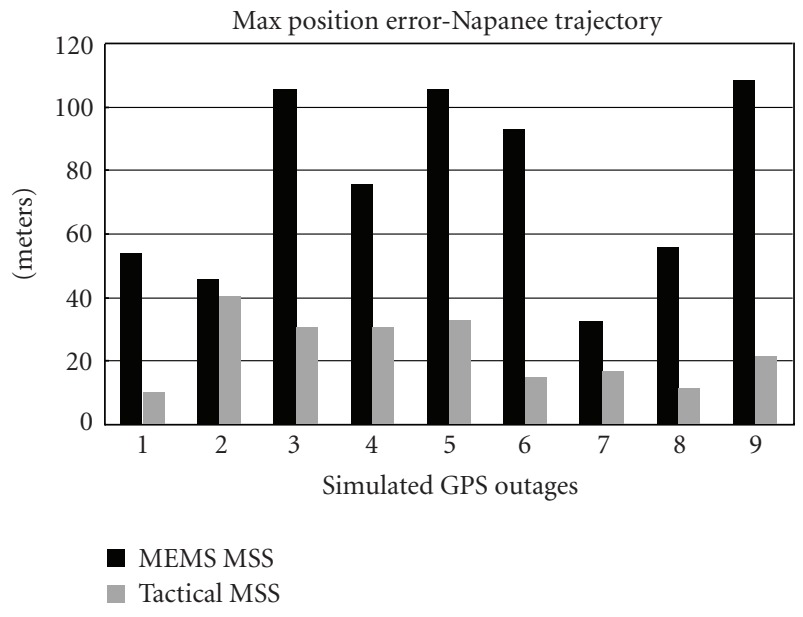

FIGURE 12: Maximum horizontal position error during nine 120second GPS outages for MEMS MSS and tactical MSS (trajectory no. 2).

position error of $12 \mathrm{~m}$ was observed with RMS of less than $8 \mathrm{~m}$ for both tactical MSS and MEMS MSS. This validates that the MSS error model was reliable and the KF operation was stable and capable of predicting the MSS errors during GPS outages. The green line showing the tactical MSS is nearly overlapping the reference solution, whereas MEMS MSS also follows the reference very closely as shown in blue.

The largest position errors for this trajectory were observed for GPS outage no. 9. This GPS outage was challenging as it consisted of a turn on a steep downward slope and detailed statistics of the outage are shown in Figure 9. It can be observed that the vehicle speed varied between 7 and $37 \mathrm{~km} / \mathrm{h}$ during this GPS outage. The acceleration and deceleration experienced during this GPS outage influenced the performance of the MSS method. The roll angle kept on varying throughout the GPS outage. Moreover, the vehicle was driven on a steep climb towards relatively horizontal plane moved downhill on a gradual slope. This can be observed from the pitch angle pattern shown in

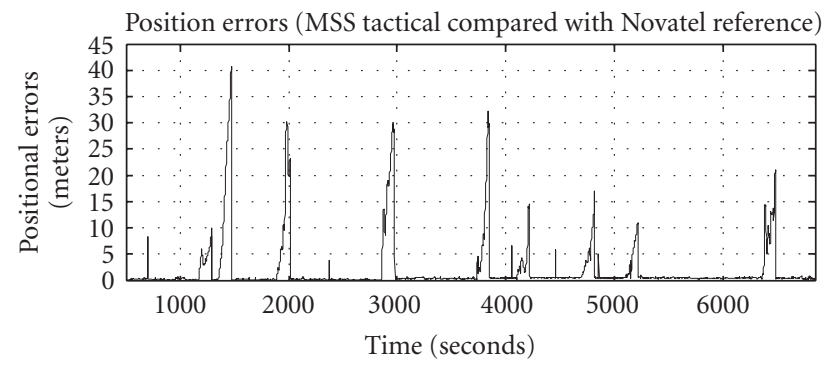

Figure 13: 2D position errors for tactical grade MSS during the outages (trajectory no. 2).

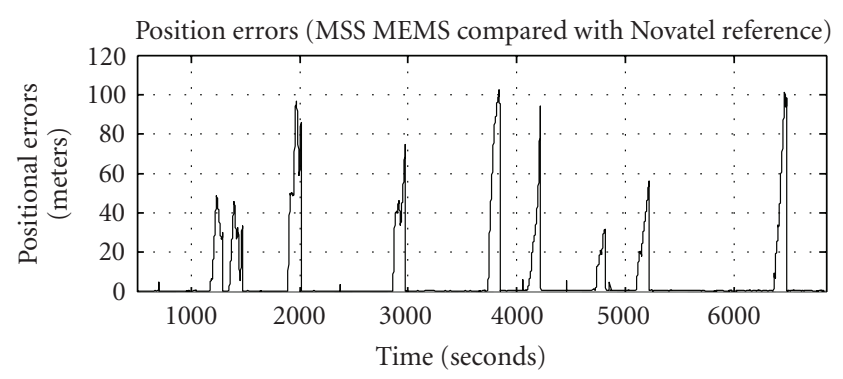

FIGURE 14: 2D position errors for MEMS grade MSS during the outages (trajectory no. 2).

Figure 9, which means that the vehicle was off the horizontal plane that was the main assumption of the MSS method. These dynamic conditions influenced the accuracy of east velocity and north velocity, thus affecting the positioning accuracy.

For outage no. 9, MEMS MSS-based KF module kept the errors to $60 \mathrm{~m}$ and for tactical MSS, the errors were only 21 meter. This validates that the MSS error model was reliable and the KF operation was stable and capable of predicting the MSS errors during GPS outages. The performance of MSS is shown in Figure 10 where tactical MSS is shown as green line and MEMS MSS depicted by blue line. 
7.2. Results for Trajectory No. 2. The second road test trajectory was around the Kingston area from Kingston to Napanee and back as is shown in Figure 11. It is a mixture of urban roadways inside Ontario starting from the city of Kingston driving towards Picton, subsequently turning towards Napanee, and then returning to Kingston. This trajectory has urban roadways in Kingston and Napanee with long highway sections. In addition, the terrain varies with many hills and winding turns. This road test was performed for 105 minutes of continuous vehicle navigation and a distance of around $85 \mathrm{Km}$ was travelled.

The ultimate check for the proposed system's accuracy is during GPS signal degradation and blockage, which was simulated and intentionally introduced in postprocessing. To assess the performance of MSS, 120 seconds simulated GPS outages were interspersed throughout the length of the trajectory during the postprocessing shown as blue circles overlaid on the map in Figure 11. The intentionally introduced GPS outages are such that they encompass all conditions of a typical trip including straight portions, turns, slopes, high speed, slow speeds, and stops.

Figure 12 compares the maximum values of the position errors for MEMS MSS and tactical MSS modules for nine GPS outages of 120 seconds. It was observed that during periods of long GPS outages, adequate positioning accuracy was obtained when using the MSS module. The worst accuracy took place during the GPS outages no. 3, no. 5, and no. 9 where the maximum error reached around $106 \mathrm{~m}$ for MEMS MSS and error for tactical MSS was $30 \mathrm{~m}, 32 \mathrm{~m}$, and $21 \mathrm{~m}$, respectively. The RMS errors for GPS outages no. 3 , no. 5 , and no. 9 were $65 \mathrm{~m}, 74 \mathrm{~m}$, and $60 \mathrm{~m}$, respectively, for MEMS MSS and $16 \mathrm{~m}, 15 \mathrm{~m}$ and $11 \mathrm{~m}$, respectively, for tactical MSS. Figures 13 and 14 show the actual 2D positional errors for both tactical and MEMS grade MSSs during the outages, respectively.

The worst performance for MEMS MSS was for three GPS outages no. 3 , no. 5 , and no. 9 where the maximum error reached around $106 \mathrm{~m}$. Figures 15 and 16 show the sections of the trajectory during outage no. 9 and outage no. 5. Figure 15 shows that outage no. 9 encountered three sharp turns despite which, the position error remained less than $21 \mathrm{~m}$ for the tactical MSS and the vehicle position (shown in green) remained within the road boundaries.

But for MEMS MSS, shown in blue line, the error increased and reached its peak of $108 \mathrm{~m}$ (RMS of $60 \mathrm{~m}$ ) at the end of the outage. Again, gyroscope scale factor error is the main reason for this performance degradation. Such error is relatively larger in case of MEMS gyroscopes, which is understandable.

The highest position errors in terms of both maximum and RMS values for this trajectory were observed for GPS outage no. 5 as seen in Figure 16.

Deep analysis of this GPS outage was therefore performed on the vehicle dynamics including the east and north velocities, azimuth, and pitch and roll angles as shown in Figure 17. It can be observed that the vehicle speed varied between 0 and $53 \mathrm{~km} / \mathrm{h}$ during this outage including a complete stop. The acceleration and deceleration experienced during this GPS outage influenced the performance

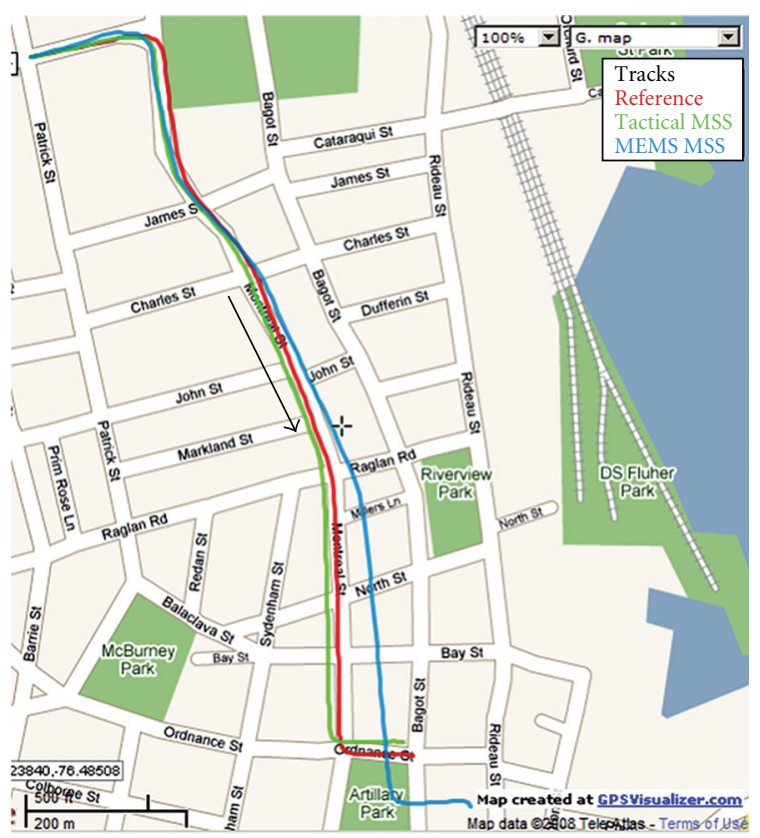

FIGURE 15: Performance during GPS outage 9 of trajectory no. 2.

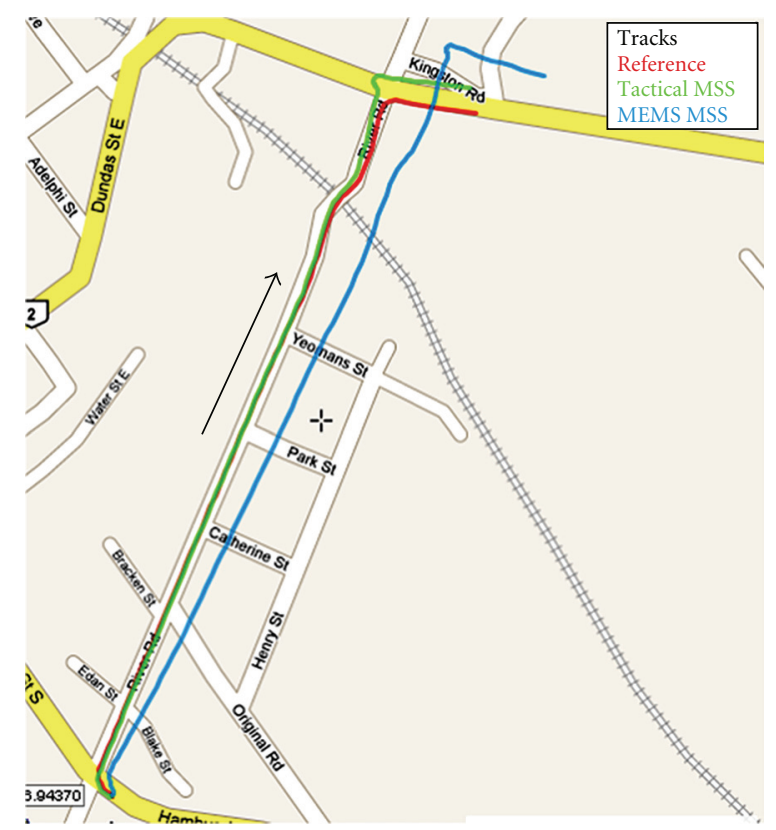

FIGURE 16: Performance during GPS outage no. 5 of trajectory no. 2.

of the MSS method. Moreover, the vehicle was driven along downhill and uphill during the same outage as can be observed from the pitch angle pattern. The pitch angle varied by 10 degrees during one section of the GPS outage. This means that the vehicle was off the horizontal plane contradicting the main assumption of the MSS method. Finally the sharp turn of the vehicle during this GPS outage may also influence the accuracy of east velocity and north velocity thus affecting the positioning accuracy. 


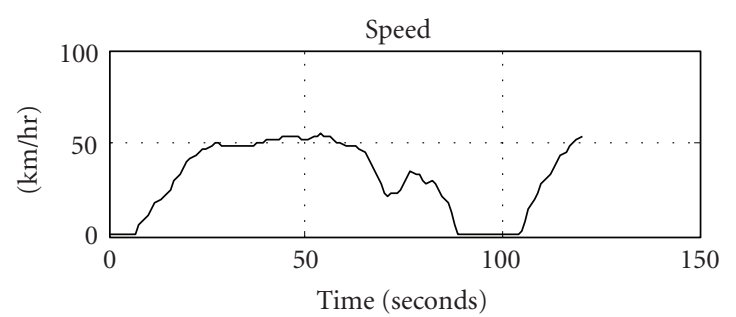

(a)

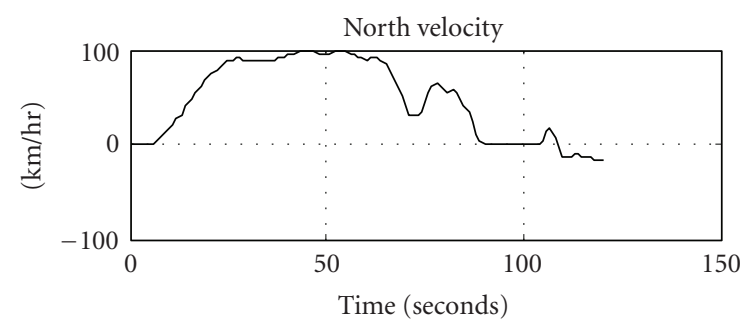

(c)

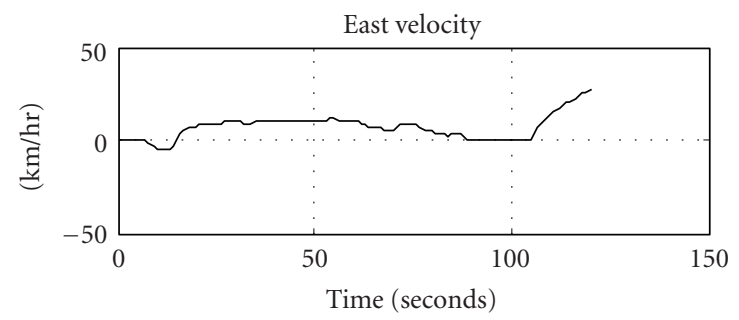

(e)

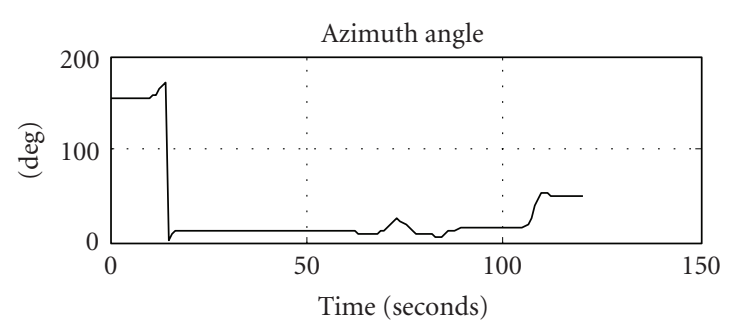

(b)

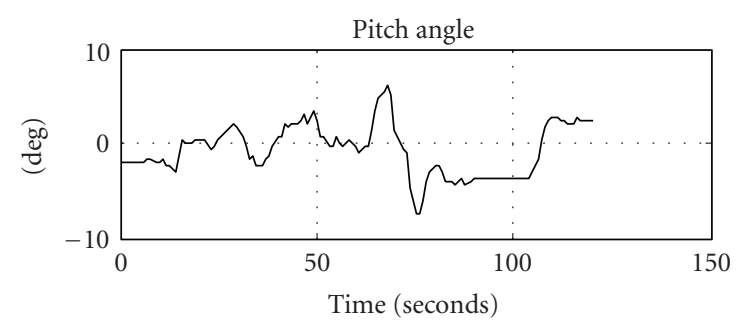

(d)

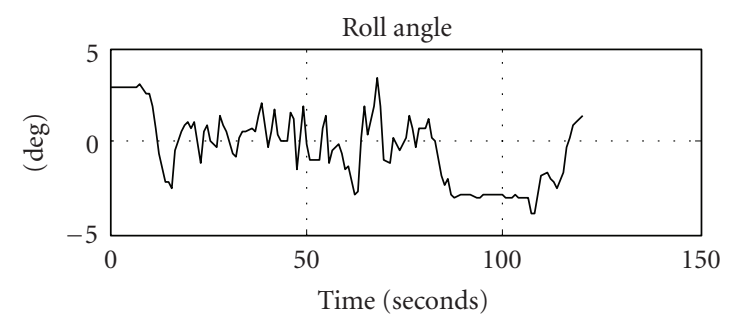

(f)

FIGURE 17: Details of vehicle dynamics during GPS outage no. 5 of trajectory no. 2.

TABLE 3: Duration of natural GPS outages in seconds for trajectory no. 3.

\begin{tabular}{lccccccccc}
\hline Outage No & 1 & 2 & 3 & 4 & 5 & 6 & 7 & 8 & 9 \\
\hline Duration $(\mathrm{sec})$ & 350 & 95 & 172 & 65 & 44 & 36 & 425 & 100 & 38 \\
\hline
\end{tabular}

This trajectory evidently proves that long duration of GPS outages, high speed, and presence of turns, changes pitch angle, and roll angle seriously affect the performance of MEMS MSS. The performance of tactical MSS shown by green line remained very stable even with all these challenges due to accurate gyroscope used.

7.3. Results for Trajectory No. 3. The last road test trajectory considered for this research was conducted in downtown Toronto as shown in Figure 18.

It was a very challenging trajectory as several natural GPS signal outages were experienced due to the presence of high rise buildings. These outages ranged from 35 seconds to as large as 425 seconds and were depicted in Figure 18 with blue circles. These outages are real nuisance to GPS-only users as they are unable to navigate properly and cannot trust GPS.

The GPS outages are such that they encompass all conditions of a typical trip including bridges, tunnels, straight portions, turns, slopes, high speed, slow speeds, and stops. This trajectory lasted for 91 minutes and a distance of around $55 \mathrm{Km}$ was traversed. The trajectory demonstrated the most realistic scenario in terms of varying length natural GPS outages. The duration of these outages is given in Table 3. The largest duration of the outage is 425 seconds and it was observed on Young street.

Figure 19 compares the maximum values of the position errors for MEMS MSS and tactical MSS for the nine GPS outages of varying lengths. It is observed that even during the periods of longer GPS outages, adequate positioning accuracy was obtained when using the MSS module. The worst accuracy occurred during the 1st GPS outages where the maximum error reached $82 \mathrm{~m}$ for MEMS MSS whereas error for tactical MSS for the same outage was $42 \mathrm{~m}$.

Both MEMS and tactical MSSs show accuracy improvement during GPS outages in all the three trajectories for various scenarios as compared to GPS alone. Performance of MEMS grade was comparable to tactical MSS when vehicle was moving on relatively straight road with slow speed during the outages. This is evident in outages $3,4,5$, and 6 where errors are around 18 meters showing that both systems can perform satisfactorily with a difference of few meters. This is due to the fact that, in these scenarios, the 


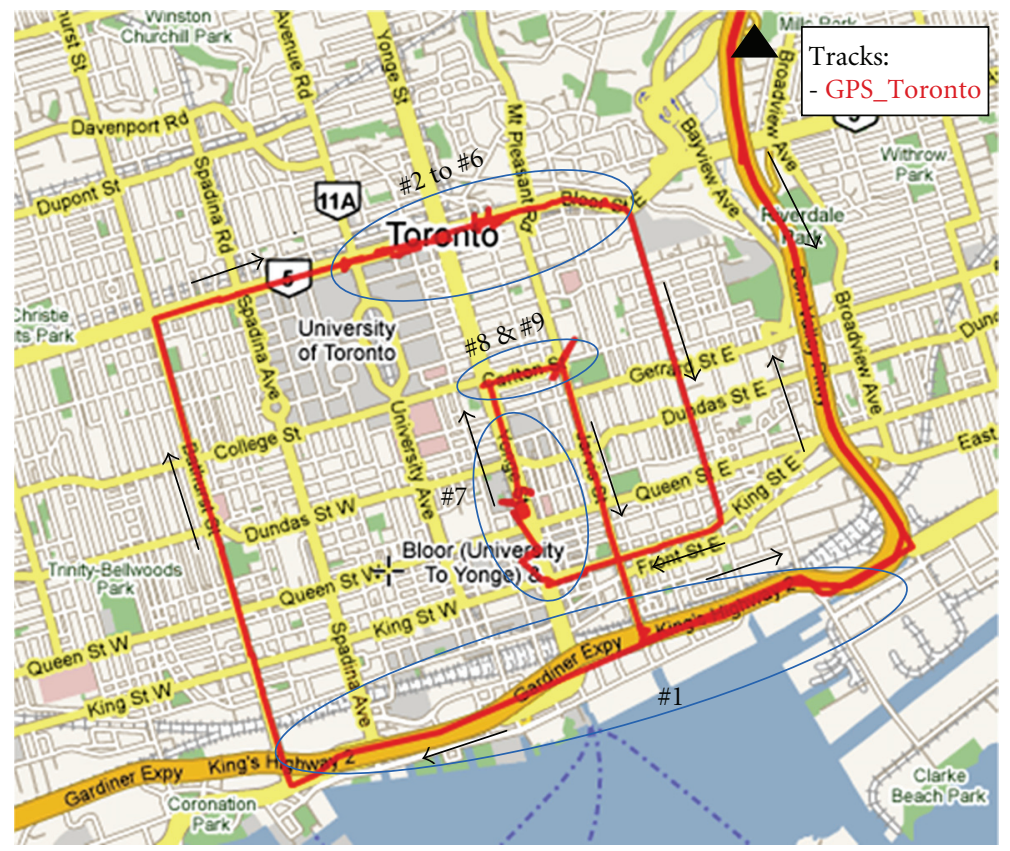

FIGURE 18: Map of trajectory no. 3 with location of nine GPS outages circled.

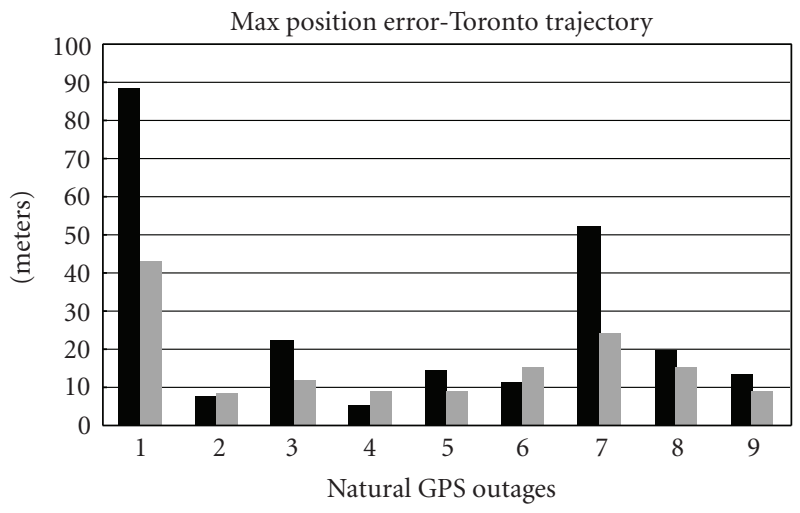

- MEMS MSS Tactical MSS

FIGURE 19: Maximum horizontal position error during nine natural GPS outages for MEMS MSS and tactical MSS (trajectory no. 3).

odometer has the high influence on the overall accuracy. However, during the high dynamic conditions, such as high speed, turns, pronounced pitch and roll angles, gyroscope plays a vital role and tactical grade MSS shows much better accuracy. This is evident in the first and seventh outages of 3rd trajectory. This difference is also noticeable throughout in 2nd trajectory.

The worst performance of the MEMS MSS was in outage no. 1. The vehicle was travelling under Gardiner Express Tunnel and its dynamics are depicted in Figure 20. In this outage the vehicle was moving in highway regions at relatively higher speeds that varied between 0 and $60 \mathrm{~km} / \mathrm{h}$ and included two complete stops. Velocity east and velocity north show rapidly changing trends causing high acceleration and deceleration which pose challenge to the performance of the MSS method. Such high speed modulated azimuth errors resulted in large position errors along the East and North directions. Maximum error of $84 \mathrm{~m}$ with RMS of $38 \mathrm{~m}$ was observed for MEMS MSS during the GPS outage of 350 seconds. With tactical MSS, maximum error grew only to about $44 \mathrm{~m}$ with RMS of 18 . The performance of MSS is shown in Figure 21. The green line shows the tactical MSS, blue line depicts MEMS MSS, and red line portrays reference solution. The outage starts from the right corner of the figure and ends at the left. It may be noticed that there are two jumps in the blue line during the outage. These jumps are caused by the sudden small turns at very low speeds which are exaggerated by the MEMS MSS due to the scale factor instability which modulates the reading of the gyroscope.

The performance of the MSS during the GPS outage no. 8 for 100 seconds is shown in Figure 21. During GPS outage no. 8 , the vehicle was on relatively straight portion of the trajectory and pitch and roll angles during the outage were small. The maximum position error of $20 \mathrm{~m}$ was observed with RMS of less than $12 \mathrm{~m}$ for both tactical MSS and MEMS MSS. This validates that the MSS error model was reliable and the KF operation was stable. Figure 22 shows the section of the trajectory during this outage where blue green line shows the tactical MSS, MEMS MSS is shown in blue, and red line shows the GPS performance.

The longest GPS outage of this trajectory was outage no. 7. Methodical analysis during this GPS outage was performed on the vehicle dynamics including the east and north velocities, azimuth, and pitch and roll angles as shown in Figure 23. 


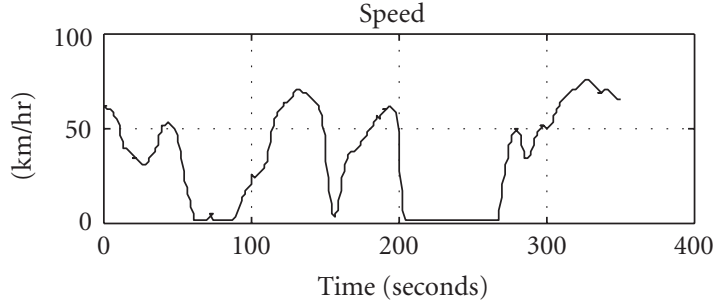

(a)

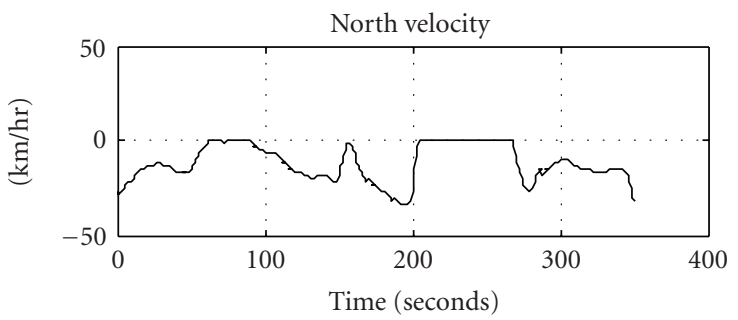

(c)

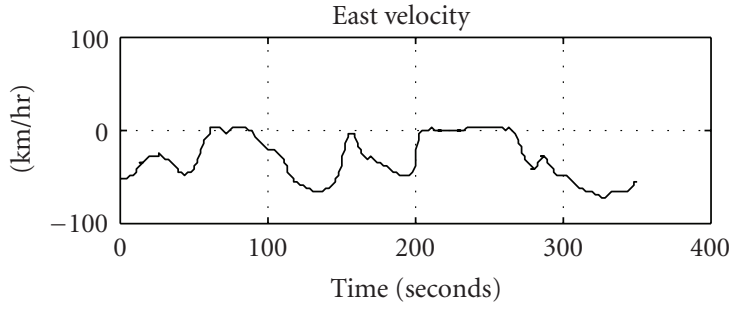

(e)

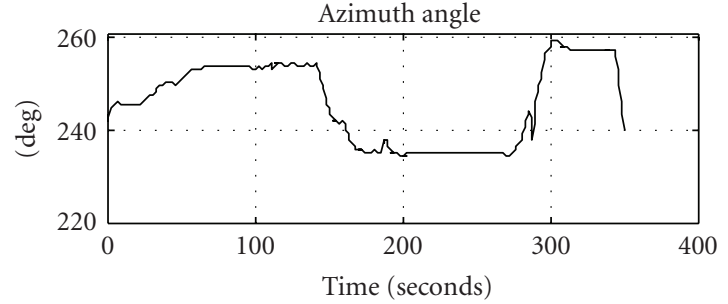

(b)

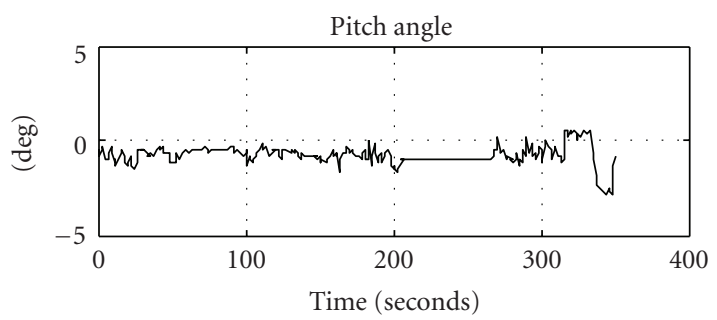

(d)

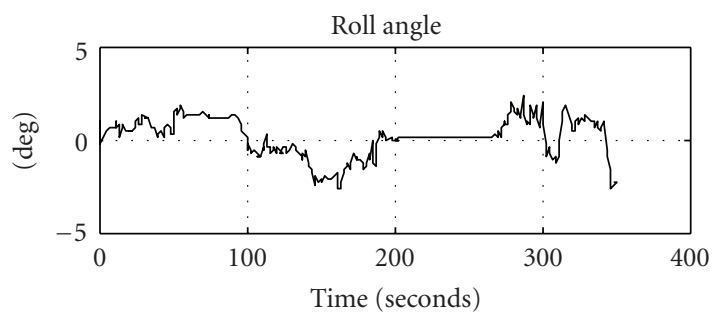

(f)

FIGURE 20: Details of vehicle dynamics during GPS outage no. 1 for 350 seconds of trajectory no. 3.

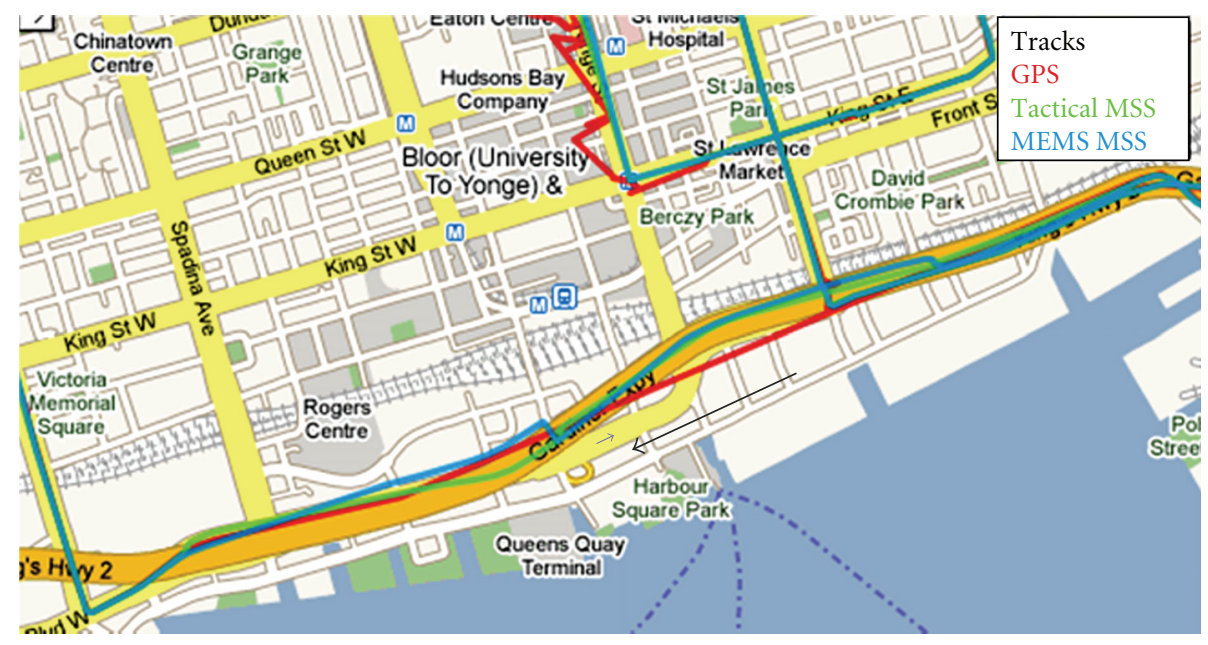

FIGURe 21: Performance during GPS outage no. 1 of trajectory no. 3.

It can be observed that, during this outage, the vehicle speed remained low and varied between 0 and $38 \mathrm{~km} / \mathrm{h}$ with several complete stops. However, with exception of one $90^{\circ}$ turn, the vehicle travelled on the straight road. Velocity east remained nearly zero after the turn for 340 seconds but velocity north showed considerable activity. However, despite the sharp turn and sudden velocity changes, maximum positional error did not exceed $30 \mathrm{~m}$ with RMS of about $21 \mathrm{~m}$ for MEMS MSS. For the tactical MSS, the maximum position error was $24 \mathrm{~m}$ with RMS value of $12 \mathrm{~m}$. The section of the trajectory during this outage is shown in Figure 24 where green line of tactical MSS remained within the road boundaries and similar performance was achieved for MEMS MSS, shown as blue line. 


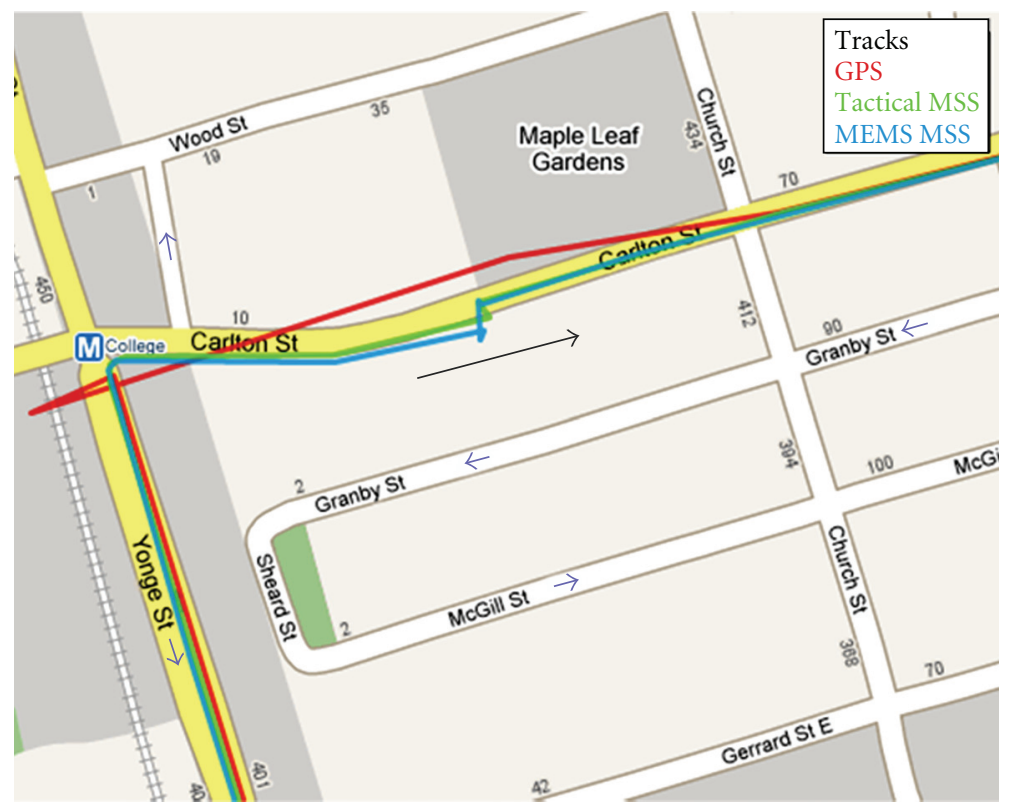

FIgURE 22: Performance during GPS outage no. 8 of trajectory no. 3.

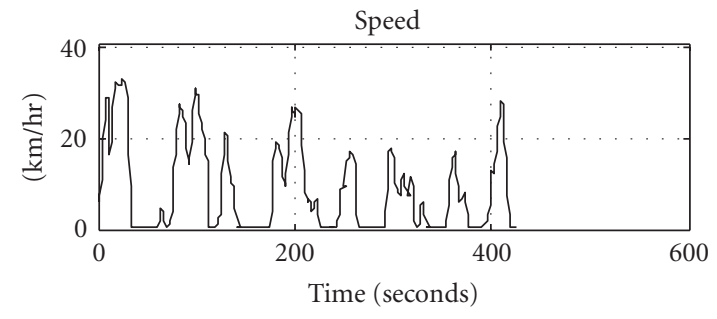

(a)

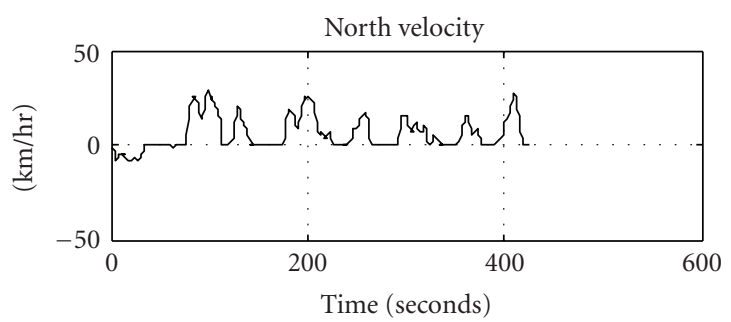

(c)

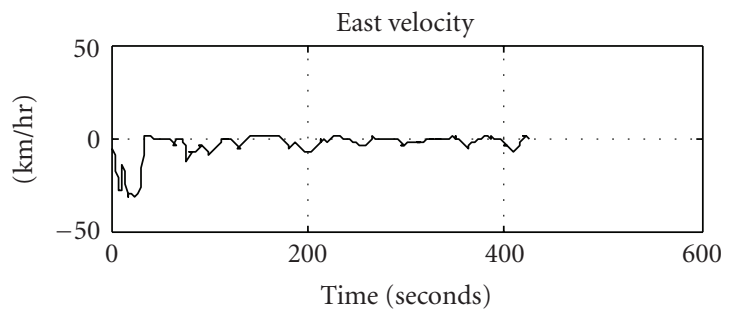

(e)

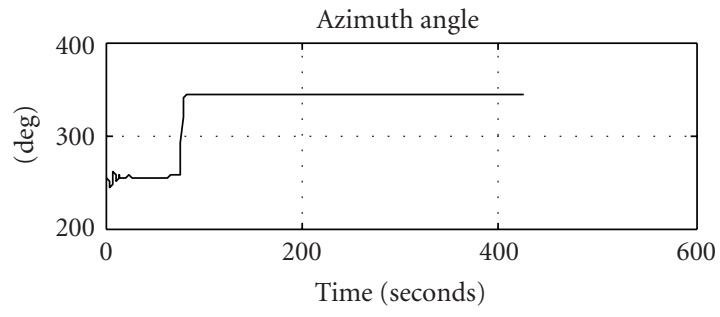

(b)

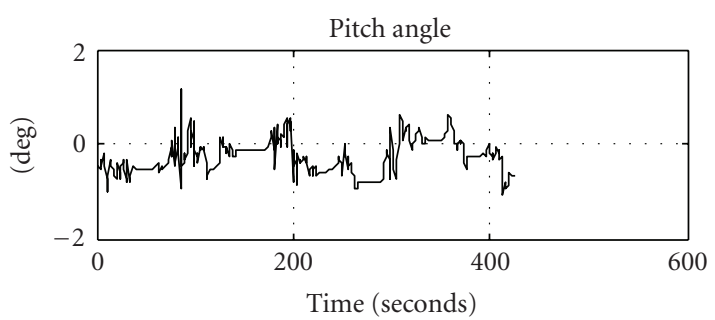

(d)

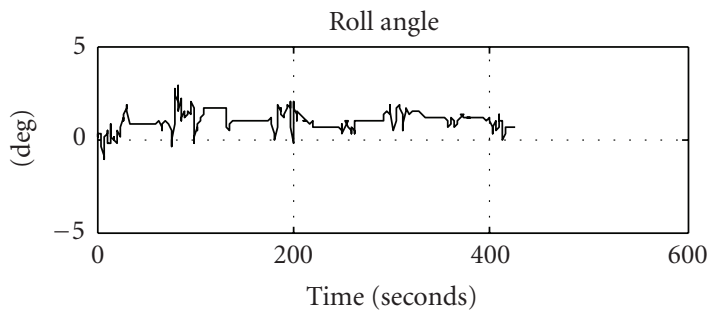

(f)

FIGURE 23: Details of vehicle dynamics during GPS outage no. 7 for 425 seconds of trajectory no. 3 . 


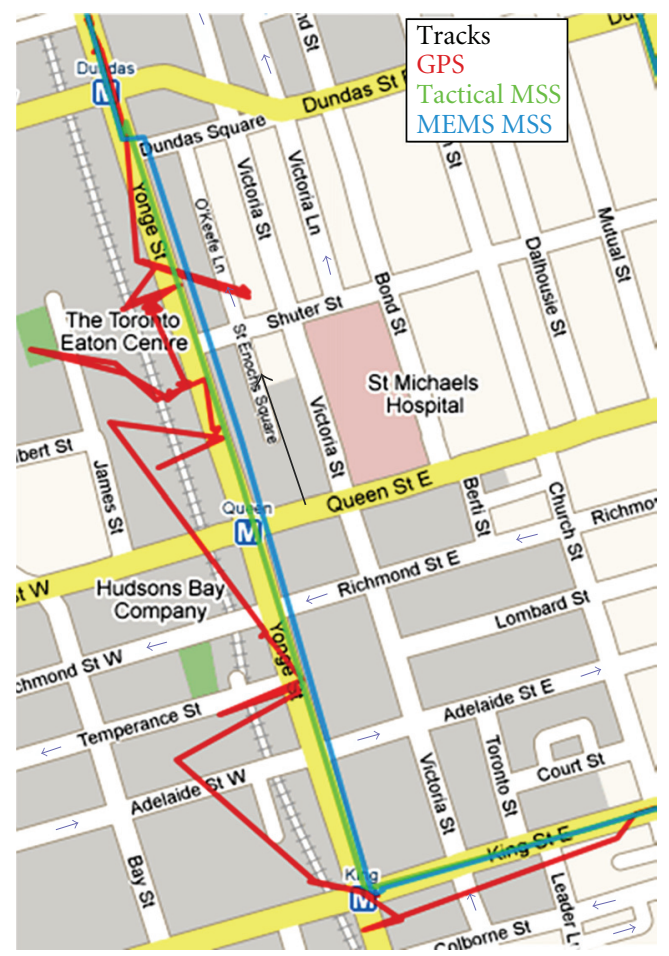

FIGURe 24: Performance during GPS outage no. 7 of trajectory no. 3.

\section{Conclusion}

This study suggested a smaller, low-cost positioning system for land applications involving reduced number of inertial sensors augmented with the measurements of the vehicle odometer and integrated with GPS. The multisensor integration module relied on $\mathrm{KF}$ which provided optimal estimation of errors through loosely coupled open-loop integration. The MSS/GPS module assumed 2D positioning in the horizontal plane and computed the vehicle heading and position. During GPS outages, KF relied on the MSS error model to predict and remove the errors and provide reliable positioning solutions. The algorithm was tested on three real-life trajectories encompassing various scenarios typically encountered on a road trip. The proposed method demonstrated promising results during short as well as longer simulated GPS outages in all the trajectories for both tactical grade and MEMS grade IMUs. The results validated that MSS/GPS integration outperformed the GPSonly system in terms of positioning solution accuracy, availability, and reliability for variety situations in any trajectory. At times, the performance of MEMS MSS/GPS system is shown to be comparable to performance of tactical MSS/GPS except when the vehicle is moving in highly dynamic conditions with very long GPS outages. The results presented in this work strongly corroborate the potential of MEMS MSS for use in land vehicle applications such as emergency applications, in-car positioning applications, fleet management system, and other location-based services applications.

\section{References}

[1] A. El-Rabbany, Introduction to GPS: The Global Positioning System, Artech House, Norwood, Mass, USA, 2002.

[2] E. D. Kaplan and C. J. Hegarty, Understanding GPS Principles and Applications, Artech House, Norwood, Mass, USA, 2nd edition, 2006.

[3] R. V. C. Wong, K. P. Schwarz, and M. E. Cannon, "Highaccuracy kinematic positioning by GPS-INS," Navigation: Journal of the Institute of Navigation, vol. 35, no. 2, pp. 275287, 1998.

[4] M. S. Grewal, L. R. Weill, and A. P. Andrews, Global Positioning Systems, Inertial Navigation, and Integration, WileyInterscience, Hoboken, NJ, USA, 2nd edition, 2007.

[5] J. A. Farrell, The Global Positioning System \& Inertial Navigation, McGraw-Hill, New York, NY, USA, 1998.

[6] R. G. Brown and P. Y. C. Hwang, Introduction to Random Signals and Applied Kalman Filtering: With MATLAB Exercises and Solutions, John Wiley \& Sons, New York, NY, USA, 3rd edition, 1997.

[7] M. S. Grewal and A. P. Andrews, Kalman Filtering: Theory and Practice Using MATLAB, John Wiley \& Sons, New York, NY, USA, 2nd edition, 2001.

[8] G. Minkler and J. Minkler, Theory and Application of Kalman Filtering, Magellan Book, Palm Bay, Fla, USA, 1993.

[9] D. Simon, "Kalman filtering," Embedded System Programming, vol. 14, no. 6, pp. 72-79, 2001.

[10] P. Zarchan, Fundamental of Kalman Filtering: A Practical Approach, AIAA, Orlando, Fla, USA, 2nd edition, 2005.

[11] R. Toledo-Moreo, M. A. Zamora-Izquierdo, B. ÚbedaMiñarro, and A. F. Gómez-Skarmeta, "High-integrity IMM-EKF-based road vehicle navigation with lowcost GPS/SBAS/INS," IEEE Transactions on Intelligent Transportation Systems, vol. 8, no. 3, pp. 491-511, 2007.

[12] G. Welch and G. Bishop, An Introduction to the Kalman Filter, ACM, Anaheim, Calif, USA, 2001.

[13] Y. Bar-Shalom, X. R. Li, and T. Kirubarajan, Estimation with Applications to Tracking and Navigation, John Wiley \& Sons, New York, NY, USA, 2001.

[14] J. Cao, S. Chen, Y. Li, and A. R. C. Ren Chongyu, "Design of integrated navigation system based on information fusion technology for the intelligent transportation system," in Proceedings of the 6th International Conference on ITS Telecommunications (ITST'06), pp. 1248-1251, Chengdu, China, June 2006.

[15] N. El-Sheimy, "The potential of partial IMUs for land vehicle navigation," Inside GNSS, vol. 3, no. 3, pp. 16-25, 2008.

[16] J.-H. Chen, S.-C. Lee, and D. B. DeBra, "Gyroscope free strapdown inertial measurement unit by six linear accelerometers," Journal of Guidance, Control, and Dynamics, vol. 17, no. 2, pp. 286-290, 1994.

[17] K. S. Mostov, A. A. Soloviev, and T.-K. J. Koo, "Accelerometer based gyro-free multi-sensor generic inertial device for automotive applications," in Proceedings of IEEE International Conference on Intelligent Transportation Systems (ITSC '97), pp. 1047-1052, Boston, Mass, USA, November 1997.

[18] S. Park and C.-W. Tan, "GPS-aided gyroscope-free inertial navigation systems," Tech. Rep. UCB-ITS-PRR-2002-22, University of California at Berkeley, Berkeley, Calif, USA, June 2002.

[19] K. Parsa, J. Angeles, and A. K. Misra, "Attitude calibration of an accelerometer array," in Proceedings of IEEE International Conference on Robotics and Automation (ICRA '02), vol. 1, pp. 129-134, Washington, DC, USA, May 2002. 
[20] Y. K. Peng and M. F. Golnaraghi, "A vector-based gyro-free inertial navigation system by integrating existing accelerometer network in a passenger vehicle," in Proceedings of IEEE/ION Position, Location and Navigation Symposium (PLANS '04), pp. 234-242, Monterey, Calif, USA, April 2004.

[21] C. A. Scott, "Improved GPS positioning for motor vehicles through map matching," in Proceedings of the 7th International Technical Meeting of the Satellite Division of the Institute of Navigation (ION GPS '94), vol. 2, pp. 1391-1400, Salt Lake City, Utah, USA, September 1994.

[22] G. Dissanayake, S. Sukkarieh, E. Nebot, and H. DurrantWhyte, "The aiding of a low-cost strapdown inertial measurement unit using vehicle model constraints for land vehicle applications," IEEE Transactions on Robotics and Automation, vol. 17, no. 5, pp. 731-747, 2001.

[23] A. Brandt and J. F. Gardner, "Constrained navigation algorithms for strapdown inertial navigation systems with reduced set of sensors," in Proceedings of the American Control Conference (ACC '98), pp. 1848-1852, Philadelphia, Pa, USA, June 1998.

[24] J.-H. Wang, Intelligent MEMS INS/GPS integration for land vehicle navigation, Ph.D. dissertation, Department of Geomatics Engineering, University of Calgary, Calgary, Canada, 2006.

[25] U. Iqbal, A. F. Okou, and A. Noureldin, "An integrated reduced inertial sensor system-RISS/GPS for land vehicle," in Proceedings of IEEE/ION Position, Location and Navigation Symposium (PLANS '08), pp. 1014-1021, Monterey, Calif, USA, May 2008.

[26] H.-J. von der Hardt, P. Arnould, D. Wolf, and M. Dufaut, "A method of mobile robot localisation by fusion of odometric and magnetometric data," The International Journal of Advanced Manufacturing Technology, vol. 9, no. 1, pp. 65-69, 1994.

[27] E. Abbott and D. Powell, "Land-vehicle navigation using GPS," Proceedings of the IEEE, vol. 87, no. 1, pp. 145-162, 1999.

[28] T. W. Lezniak, R. W. Lewis, and R. A. McMillen, "A dead reckoning/map correlation system for automatic vehicle tracking," IEEE Transactions on Vehicular Technology, vol. 26, no. 1, pp. 47-60, 1977.

[29] A. Gelb, Applied Optimal Estimation, The MIT Press, Cambridge, Mass, USA, 1974.

[30] L. D. Hostetler and R. D. Andreas, "Nonlinear Kalman filtering techniques for terrain-aided navigation," IEEE Transactions on Automatic Control, vol. 28, no. 3, pp. 315-323, 1983.

[31] D. Titterton and J. Weston, Strapdown Inertial Navigation Technology, AIAA, Orlando, Fla, USA, 2nd edition, 2005.

[32] K. R. Britting, Inertial Navigation System Analysis, John Wiley \& Sons, New York, NY, USA, 1971.

[33] A. Noureldin, EE523: Course Notes on Mobile Multi-Sensor Systems Integration, Royal Military College, Kingston, Canada, 2007.

[34] A. Papoulis and S. U. Pillai, Probability, Random Variables, and Stochastic Processes, McGraw-Hill, New York, NY, USA, 2002.

[35] P. S. Maybeck, Stochastic Models, Estimation, and Control. Vol. 1, Academic Press, New York, NY, USA, 1979.

[36] G. Welch and G. Bishop, "An introduction to the Kalman filter," in Proceedings of the 28th Annual Conference on Computer Graphics and Interactive Techniques (SIGGRAPH '01), Los Angeles, Calif, USA, August 2001.

[37] M. Hayes and J. Treichler, "Adaptive filtering," IEEE Signal Processing Magazine, vol. 25, no. 6, pp. 169-172, 2008.

[38] J. Dah-Jing and W. Tsu-Pin, "An adaptive sensor fusion method with applications in integrated navigation," Journal of Navigation, vol. 61, no. 4, pp. 705-721, 2008.
[39] Z.-L. Deng and G. Hao, "Self-tuning multisensor measurement fusion Kalman estimator and its convergence analysis," Control Theory and Applications, vol. 25, no. 5, pp. 845-852, 2008.

[40] J. Bernstein, "An overview of MEMS inertial sensing technology," Sensors Magazine, February 2003.

[41] CarChip E/X OBDII-Based Vehicle Data Logger and Software, September 2008, http://www.davisnet.com/product_ documents/drive/spec_sheets/8211-21-25_carchip_specsB.pdf.

[42] "SPAN Technology User Manual," OM-20000062, NovAtel Inc. 

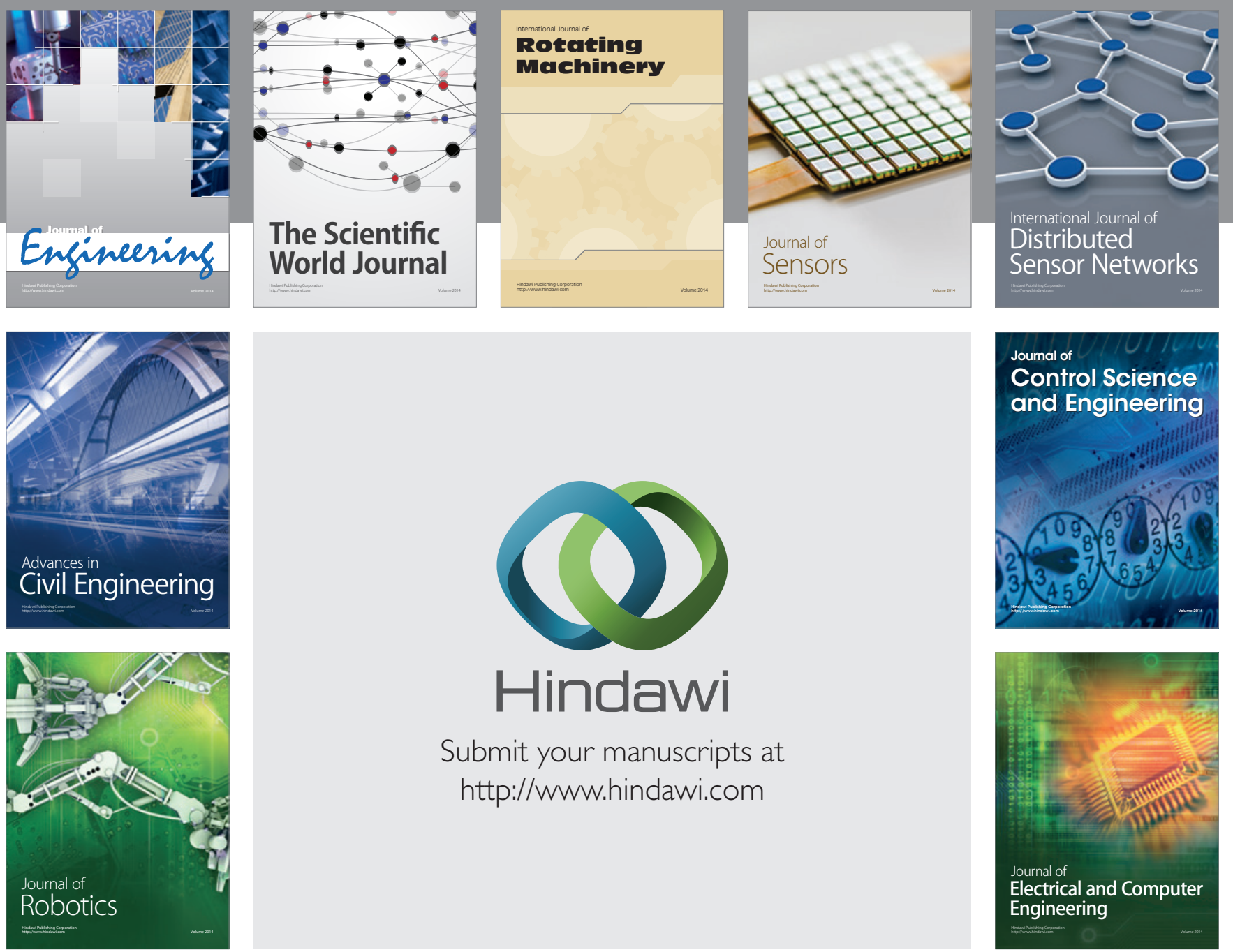

Submit your manuscripts at

http://www.hindawi.com
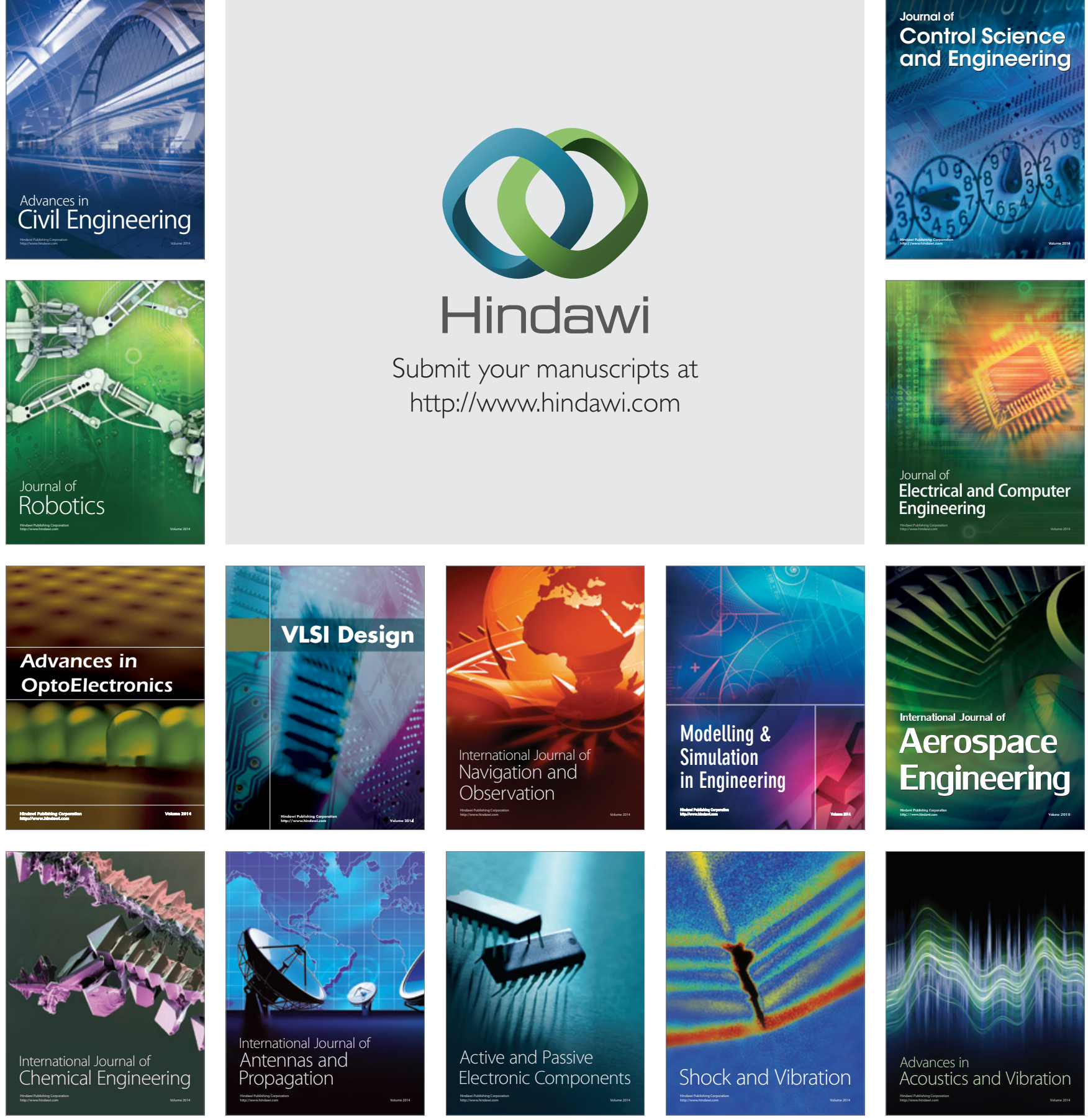